\title{
The COPII cargo adapter SEC24C is essential for neuronal homeostasis
}

\author{
Bo Wang, ${ }^{1,2}$ Joung Hyuck Joo, ${ }^{1,2}$ Rebecca Mount, ${ }^{1,2}$ Brett J. W. Teubner, ${ }^{3}$ Alison Krenzer, ${ }^{1,2}$ Amber L. Ward, ${ }^{1,2}$ Viraj P. Ichhaporia, ${ }^{4}$ \\ Elizabeth J. Adams, ${ }^{5}$ Rami Khoriaty, ${ }^{6}$ Samuel T. Peters, ${ }^{2,7}$ Shondra M. Pruett-Miller, ${ }^{2,7}$ Stanislav S. Zakharenko, ${ }^{3}$ \\ David Ginsburg, ${ }^{5,6,8}$ and Mondira Kundu, ${ }^{1,2}$ \\ 'Department of Pathology, ${ }^{2}$ Department of Cell and Molecular Biology, ${ }^{3}$ Department of Developmental Neurobiology, and ${ }^{4}$ Department of Tumor Cell Biology, St. Jude Children's Research Hospital, Memphis,

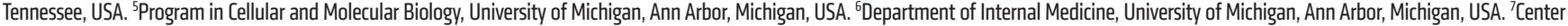 \\ for Advanced Genome Engineering, St. Jude Children's Research Hospital, Memphis, Tennessee, USA. ${ }^{8}$ Howard Hughes Medical Institute, Life Sciences Institute, and Departments of Human Genetics and \\ Pediatrics, University of Michigan, Ann Arbor, Michigan, USA.
}

SEC24 family members are components of the coat protein complex II (COPII) machinery that interact directly with cargo or with other adapters to ensure proper sorting of secretory cargo into COPII vesicles. SEC24C is 1 of 4 mammalian SEC24 paralogs (SEC24A-D), which segregate into 2 subfamilies on the basis of sequence homology (SEC24A/SEC24B and SEC24C/ SEC24D). Here, we demonstrate that postmitotic neurons, unlike professional secretory cells in other tissues, are exquisitely sensitive to loss of SEC24C. Conditional KO of Sec24c in neural progenitors during embryogenesis caused perinatal mortality and microcephaly, with activation of the unfolded protein response and apoptotic cell death of postmitotic neurons in the murine cerebral cortex. The cell-autonomous function of SEC24C in postmitotic neurons was further highlighted by the loss of cell viability caused by disrupting Sec24c expression in forebrain neurons of mice postnatally and in differentiated neurons derived from human induced pluripotent stem cells. The neuronal cell death associated with Sec24c deficiency was rescued in knockin mice expressing Sec24d in place of Sec24c. These data suggest that SEC24C is a major cargo adapter for COPIIdependent transport in postmitotic neurons in developing and adult brains and that its functions overlap at least partially with those of SEC24D in mammals.

\section{Introduction}

ER-to-Golgi trafficking of secretory cargoes, which comprise nearly one-third of the mammalian proteome, is a highly conserved process that helps maintain cellular homeostasis and function. Defects in ER-to-Golgi trafficking have been associated with various diseases, including neurodegeneration (1). In patients with Parkinson's disease, for example, defects in ER-to-Golgi trafficking are thought to contribute to cytotoxicities associated with the abnormal expression of $\alpha$-synuclein, a major component of Lewy bodies (2). Although the basic molecular mechanisms involved in sorting secretory cargoes into coat protein complex II-coated (COPII-coated) vesicles destined for the Golgi are well characterized $(3,4)$, the cell type-specific requirements for COPII proteins in mammals are only beginning to be explored.

SEC23-SEC24 heterodimers and SEC13-SEC31 heterotetramers form the inner and outer coats of COPII vesicles, respectively, and are recruited to the cytosolic surface of the ER by GTP-bound SAR1 $(4,5)$. These proteins drive the formation of vesicles that transport cargo to the Golgi apparatus $(4,5)$. In addition to the

Conflict of interest: The authors have declared that no conflict of interest exists Submitted: October 20, 2017; Accepted: May 8, 2018.

Reference information: J Clin Invest. 2018;128(8):3319-3332.

https://doi.org/10.1172/JCI98194. essential inner coat proteins SEC23 and SEC24, Saccharomyces cerevisiae also expresses 2 nonessential SEC24-related proteins, SFB2 and SFB3 (also known as LST1) $(6,7)$. Vertebrates express multiple paralogs of SEC23 (i.e., SEC23A and SEC23B) and SEC24 (i.e., SEC24A-D) (8); these isoforms may have evolved to accommodate the expanded repertoire of COPII cargoes required for vertebrate development and/or to allow regulated expression in a broader range of cell types. The human diseases and phenotypes in model organisms (e.g., zebrafish and mice) associated with mutations in SEC23 and SEC24 paralogs highlight some of the cell type- and context-specific functions of these genes in higher eukaryotes. For example, in humans, missense mutations in SEC23A cause cranio-lenticulo-sutural dysplasia due to defective collagen secretion $(9,10)$, and loss-of-function mutations in SEC23B cause type II congenital dyserythropoeitic anemia (11). Disruption of $\operatorname{Sec} 23 a$ or $\operatorname{Sec} 23 b$ expression in zebrafish causes defective secretion of extracellular matrix proteins and a phenotype akin to cranio-lenticulo-sutural dysplasia $(9,12)$. Zebrafish with knockdown of $\operatorname{Sec} 23 b$ also present with anemia (11), recapitulating the phenotype in humans. Sec23a deficiency in mice leads to midgestational lethality associated with intracellular accumulation of collagens in multiple tissues, defective development of extraembryonic membranes, and neural tube opening in the midbrain (13); in contrast, $\operatorname{Sec} 23 b$ deficiency leads to perinatal lethality due to pancreatic insufficiency and defects in other exocrine 
A

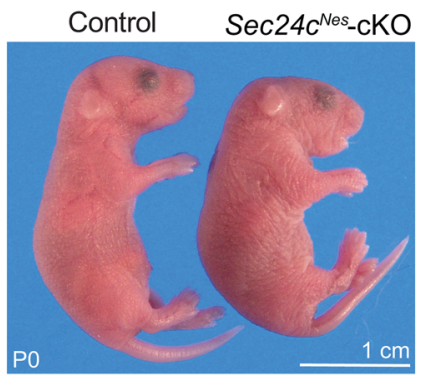

B

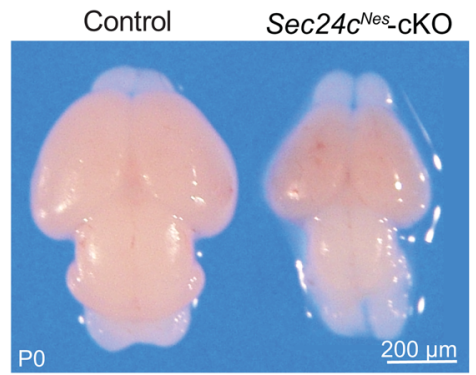

C

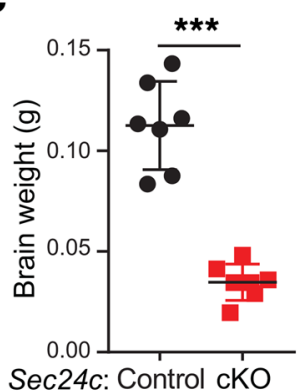

D

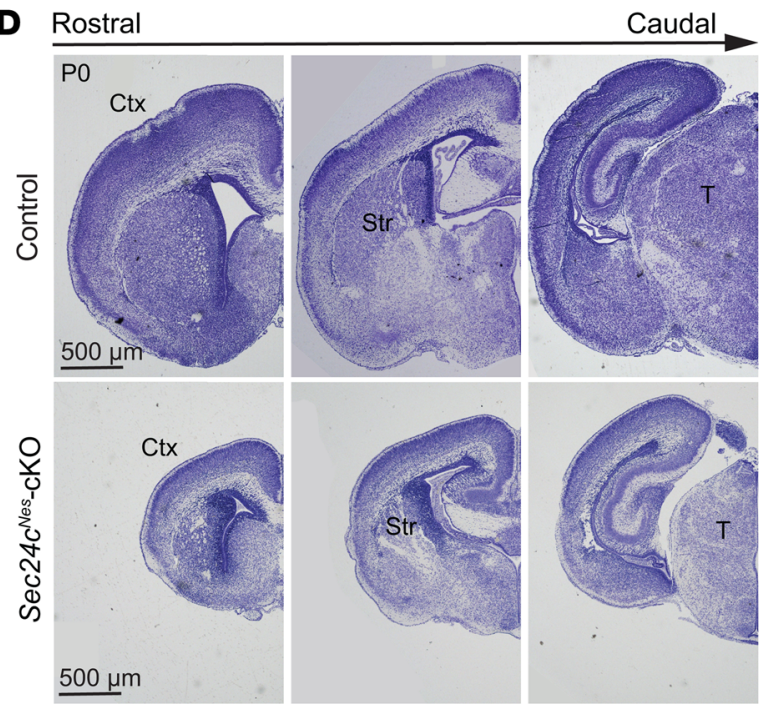

E

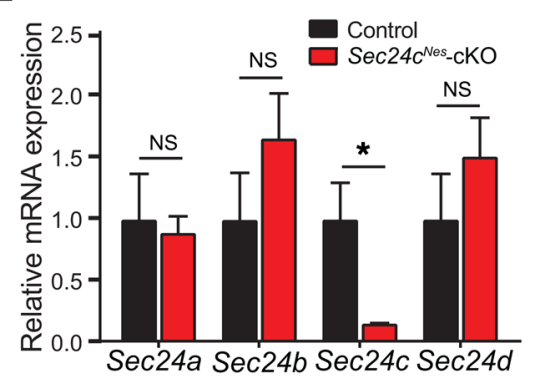

$\mathbf{F}$

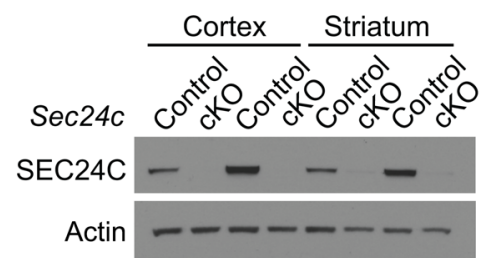

Figure 1. CNS-specific deletion of Sec24c leads to microcephaly. (A) Representative photographs of control and Sec24 $c^{\text {Nes }-c K O ~ m i c e ~ a t ~ P O . ~(B) ~ R e p r e s e n t a-~}$ tive images of dissected brains from control and Sec24 $c^{\text {Nes }}$-CKO mice. (C) Brain weights (mean \pm SEM) of Sec $24 c^{\text {Nes }}$-cKO mice $(n=7)$ and control mice $(n=7)$ at PO. (D) Nissl staining of brain sections along the rostrocaudal axis in control and Sec24cNes-cKO mice at PO. (E) Sec24a, Sec24b, Sec24c, and Sec24d mRNA levels (mean \pm SEM) in the cortices of Sec24 $C^{\text {Nes }}$ - cKO $(n=5)$ and control $(n=5)$ mice. $(\mathbf{F})$ Immunoblot analyses of cortical and striatal extracts confirming the loss of SEC24C protein in Sec24C ${ }^{\text {Nes }}$-cKO mice. $n=2$ for each genotype. Scale bars: $1 \mathrm{~cm}$ (A), $200 \mu \mathrm{m}$ (B), and $500 \mu \mathrm{m}$ (D). cKO, conditional knockout; Ctx, cortex; Str, striatum; T, thalamus. ${ }^{*} P<0.05$ and ${ }^{* *} P<0.001$, by Student's $t$ test.

glands (14). Why SEC23B mutations affect different cell types in mice and humans remains unclear but may reflect differences in the expression patterns in these species.

The existence of multiple SEC24 isoforms, which have distinct and overlapping functions, increases the diversity of proteins recruited into COPII vesicles, as each protein contains distinct cargo-binding sites with different affinities for various transport signals (3,15-20). In mammals, certain export signals interact with all 4 SEC24 isoforms, albeit with varying strengths (21), whereas other signals are more selective. For example, SEC24A and SEC24B, which share approximately $50 \%$ amino acid identity, both interact with LxxLE and DxE signals (8, 22). SEC24C and SEC24D, which are also approximately $50 \%$ identical to each other but share only $20 \%$ identity with SEC24A and SEC24B, bind an IxM-packaging signal (8, 22). Individual SEC 24 isoforms are also essential for the ER export of specific cargo. Serotonin and GABA transporters, for example, exclusively use SEC24C and SEC24D, respectively $(23,24)$.

Although SEC24A and SEC24B recognize similar binding motifs on cargo, mice lacking Sec $24 a$ or $\operatorname{Sec} 24 b$ show different deficits. Sec24a-KO mice are viable and normal, except for their reduced plasma cholesterol levels (25). Inactivation of Sec24b in mice causes midgestational (E12.5) lethality with neural tube closure defects. Loss-of-function mutations in SEC24B in humans are also associated with neural tube defects (26). The phenotype in Sec24a-KO mice is due to a defect in PCSK9 secretion (25), and that in Sec24b-KO mice is due, at least in part, to an inability of the COPII machinery to recognize and sort VANGL2, a component of the planar cell polarity-signaling pathway (27). Nevertheless, consistent with the redundancy in function between SEC24A and SEC24B, plasma cholesterol levels are reduced further in $\operatorname{Sec} 24 a$-deficient mice that are also haploinsufficient for $\operatorname{Sec} 24 b$, but not in those also haploinsufficient for Sec24d (25).

Mutant SEC24D alleles in humans are associated with an autosomal-recessive form of osteogenesis imperfecta (28-30). The role of SEC24D in bone development is also evident in Sec24d-mutant zebrafish, which show defects in secretion of extracellular matrix proteins, resulting in malformed cartilage and craniofacial dysmorphism (31). Although these deficits are specific to $S e c 24 d$ mutant animals and are not recapitulated by the loss of $\operatorname{Sec} 24 c$, the loss of both proteins accelerates the arrest in craniofacial development (31). Mice with $\operatorname{Sec} 24 c$ or $\operatorname{Sec} 24 d$ deficiency exhibit early embryonic lethality at E7.5 or E3.5, respectively $(32,33)$. 


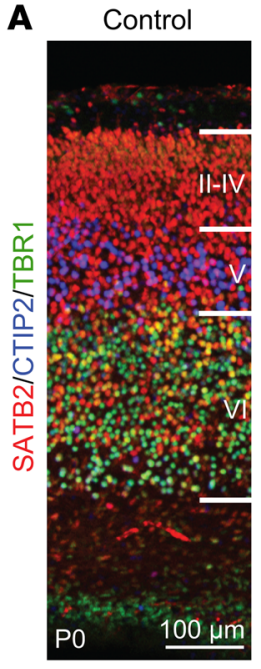

C

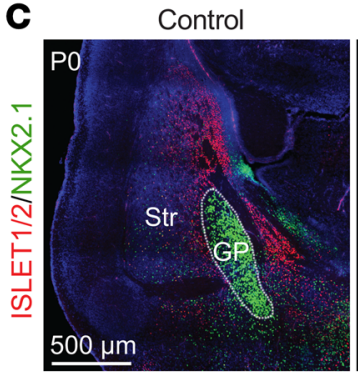

$\operatorname{Sec} 24 C^{\text {Nes-CKO }}$

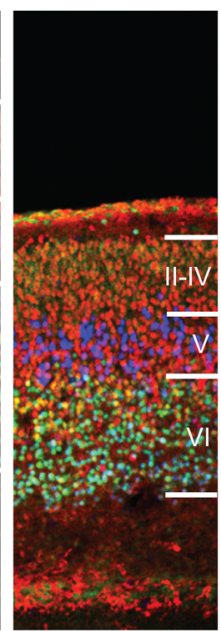

B

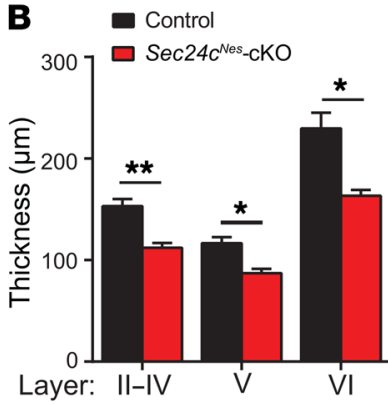

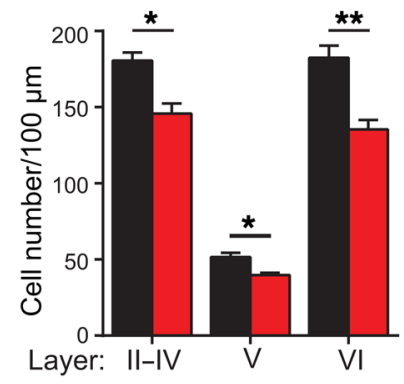
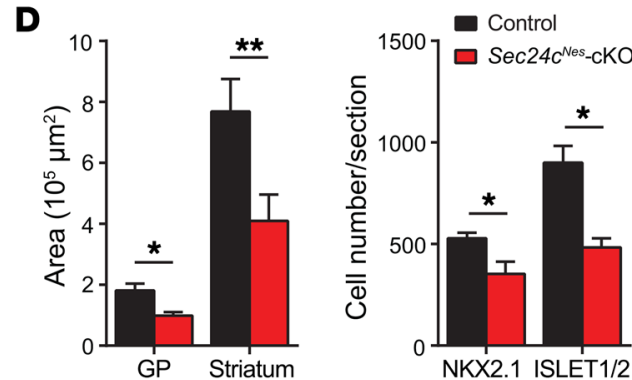

Figure 2. The brains of Sec24c-deficient mice contain fewer neurons than brains of control mice. (A) Representative images of layers II-VI in control $(n=3)$ and Sec24C Nes - cKO $(n=3)$ mice at PO, as delineated by expression of the callosal neuron marker SATB2, the layer V marker CTIP2, and the layer VI marker TBR1. Scale bar: $100 \mu \mathrm{m}$. (B) Thickness of designated cortical layers (mean \pm SEM) and numbers of neurons within those layers (mean \pm SEM) from $\operatorname{Sec} 24 c^{\text {Nes }}$ - CKO $(n=3)$ and control $(n=3)$ mice. (C) Representative images of immunostained brain sections showing ISLET1/2 ${ }^{+}$neurons in the striatum and NKX2.1+ neurons in the globus pallidus of control $(n=3)$ and Sec24c $c^{\mathrm{Nes}}-\mathrm{cKO}(n=3)$ mice at P0. Scale bar: $500 \mu \mathrm{m}$. (D) Mean area $( \pm$ SEM) covered by and mean number $\left( \pm\right.$ SEM) of ISLET1/2+ and NKX2.1+ cells in the striatum and globus pallidus of Sec $24 c^{\text {Nes}}$-cKO $(n=3)$ and control $(n=3)$ mice. GP, globus pallidus. ${ }^{*} P<0.05$ and ${ }^{*} P<0.01$, by Student's $t$ test.

Despite the importance of SEC24C during embryonic development and that of ER-to-Golgi trafficking in professional secretory cells, the tissue-specific deletion of $\operatorname{Sec} 24 \mathrm{c}$ in hepatocytes, pancreatic cells, smooth muscle cells, and intestinal epithelial cells does not cause any appreciable defects in mice (32). This finding suggests that other SEC24 paralogs compensate, at least in those cell types tested. Given that certain neurotransmitter transporters (e.g., serotonin and GABA transporters) appear to exclusively use SEC24C or SEC24D $(23,24)$, we hypothesized that neurons may be more sensitive to $\operatorname{Sec} 24 c$ deficiency than are other secretory cells. Therefore, we generated mice lacking $\operatorname{Sec} 24 c$ expression in the CNS to evaluate the function of SEC24C in neurons. We also tested the degree of functional overlap between SEC24C and SEC24D in neurons by using a recently developed murine model, in which Sec24d was knocked into the Sec24c locus (34).

\section{Results}

Sec24c deficiency in the embryonic brain leads to perinatal lethality and microcephaly. To examine the consequences of $\operatorname{Sec} 24 \mathrm{c}$ deficiency in the CNS, we crossed mice harboring a floxed Sec $24 c$ allele (32) with Nes-Cre-transgenic mice, in which the expression of Cre recombinase is driven by the Nestin gene promoter expressed specifically in neural progenitors starting at E10.5 (35). Sec2 $4 \mathrm{C}^{\nexists l f l}$ Nes-Cre mice (hereafter referred to as $\operatorname{Sec} 24 c^{\text {Nes }}$ conditional-KO [ $\mathrm{Sec} 24 \mathrm{c}^{\text {Nes-cKO}}$ ] mice) were generated by breeding $\operatorname{Sec} 24 c^{+/ f l}$ Nes-Cre mice with Sec$24 \mathrm{c}^{\text {llfl}}$ mice. Offspring were born at the expected Mendelian ratio (15 Sec $24 c^{\text {Nes-cKO}} / 58$ total mice: $25.86 \%$ ) with flattened heads (Figure 1A) and became cyanotic and died shortly after birth. Dissection of brains from mice sacrificed shortly after birth on PO revealed

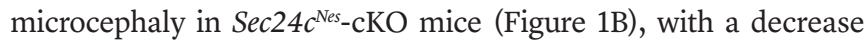
of approximately $50 \%$ in fixed brain weight compared with that of control brains (Figure 1C). Nissl staining of brain sections also highlighted the smaller size of the cortex, striatum, and thalamus in Sec$24 \mathrm{C}^{\mathrm{Nes}}$-CKO mice at PO (Figure 1D). Real-time RT-PCR and immunoblot analyses confirmed the loss of $\operatorname{Sec} 24 \mathrm{c}$ expression in the brains

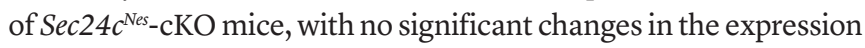
of $\operatorname{Sec} 24 a, \operatorname{Sec} 24 b$, or $\operatorname{Sec} 24 d$ (Figure 1, E and F).

SEC24C deletion triggers apoptotic loss of postmitotic neurons during embryonic development. To determine whether the microcephaly resulting from $\operatorname{Sec} 24 \mathrm{c}$ deficiency was associated with fewer neurons, we stained brain sections with antibodies against the following neuronal markers: SATB2 (callosal neurons, layers II-VI), CTIP2 (layer V), and TBR1 (layer VI) (Figure 2A and Supplemental Figure 1; supplemental material available online with this article; https://doi. 
A
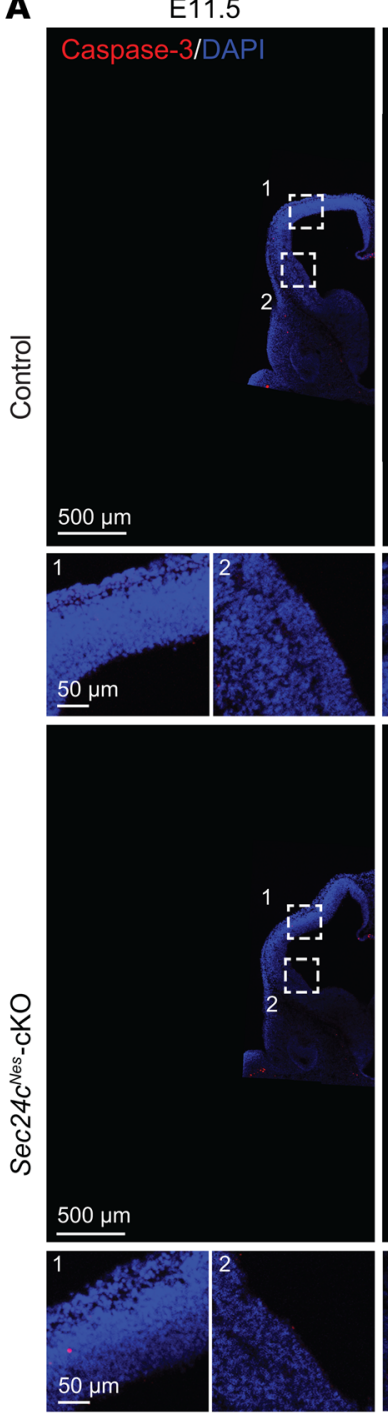

B

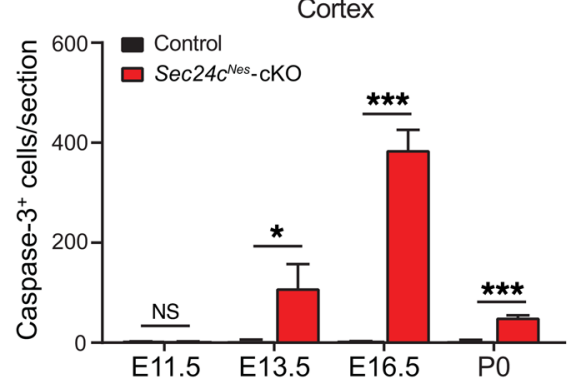

E13.5
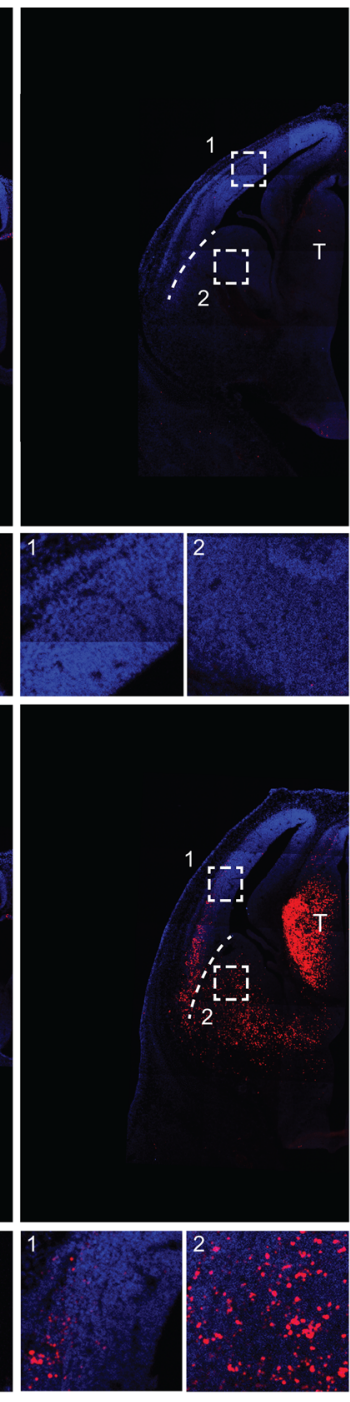

E16.5
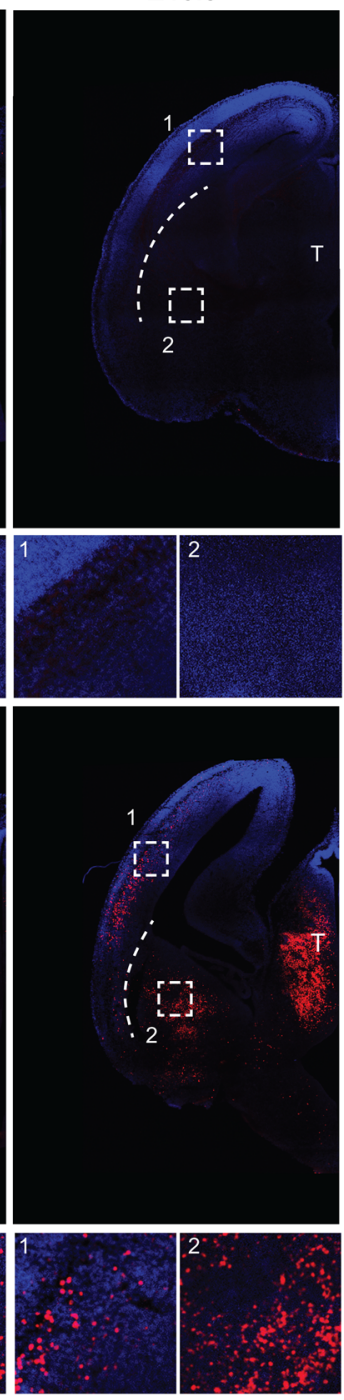

P0
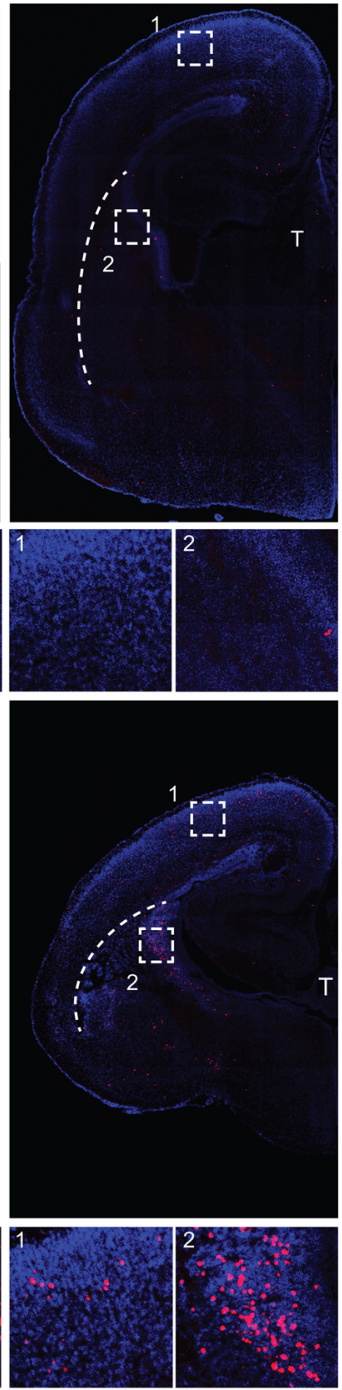

Striatum

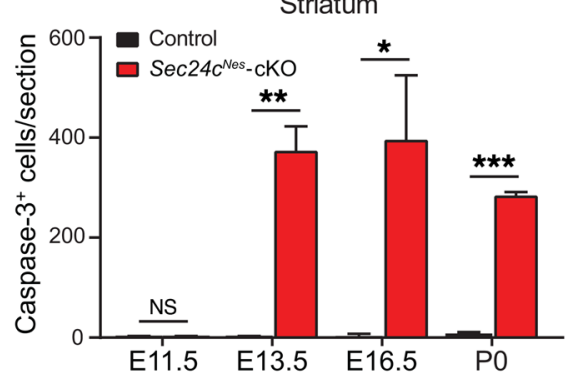

Figure 3. Sec24c deficiency leads to widespread apoptotic cell death in embryonic brains. (A) Representative images of cleaved caspase-3 staining in brains from control and Sec24 $c^{\text {Nes }}$-CKO mice at E11.5, E13.5, E16.5, and P0. Magnified areas from cortex (boxed area 1) and striatum (boxed area 2) are also shown. Scale bars: $500 \mu \mathrm{m}$ (A) and $50 \mu \mathrm{m}$ (A, insets). (B) Number (mean \pm SEM) of caspase- $3^{+}$cells in the cortex and striatum in brains from control and Sec24 $C^{\text {Nes }}$-CKO mice at E11.5, E13.5, E16.5, and P0. $n=3$ for each genotype at each age. ${ }^{*} P<0.05$, ${ }^{*} P<0.01$, and ${ }^{* * *} P<0.001$, by Student's $t$ test.

org/10.1172/JCI98194DS1). Both the thickness of individual cortical layers and the numbers of neurons in each layer were markedly less in Sec24c $c^{\text {Nes }}$-cKO mice than in controls (Figure 2B). Similarly, NKX2.1 and ISLET1/2 labeling of striatal neurons in the globus pallidus and striatum, respectively, revealed substantially reduced area and numbers of cells in Sec $24 c^{N e s}$-cKO mice (Figure 2, C and D).
To determine whether the decrease in neurons in Sec$24 c^{N e s}$ - $\mathrm{CKO}$ mice could be attributed to apoptotic cell death, we stained sections using an antibody against cleaved caspase-3. We detected cleaved caspase-3 staining in the cortex and thalamus of Sec2 $4 c^{\text {Nes }}$ - $\mathrm{CKO}$ mice beginning at E13.5, peaking at E16.5, and fading by PO (Figure 3, A and B). Cleaved caspase-3 positivity in 

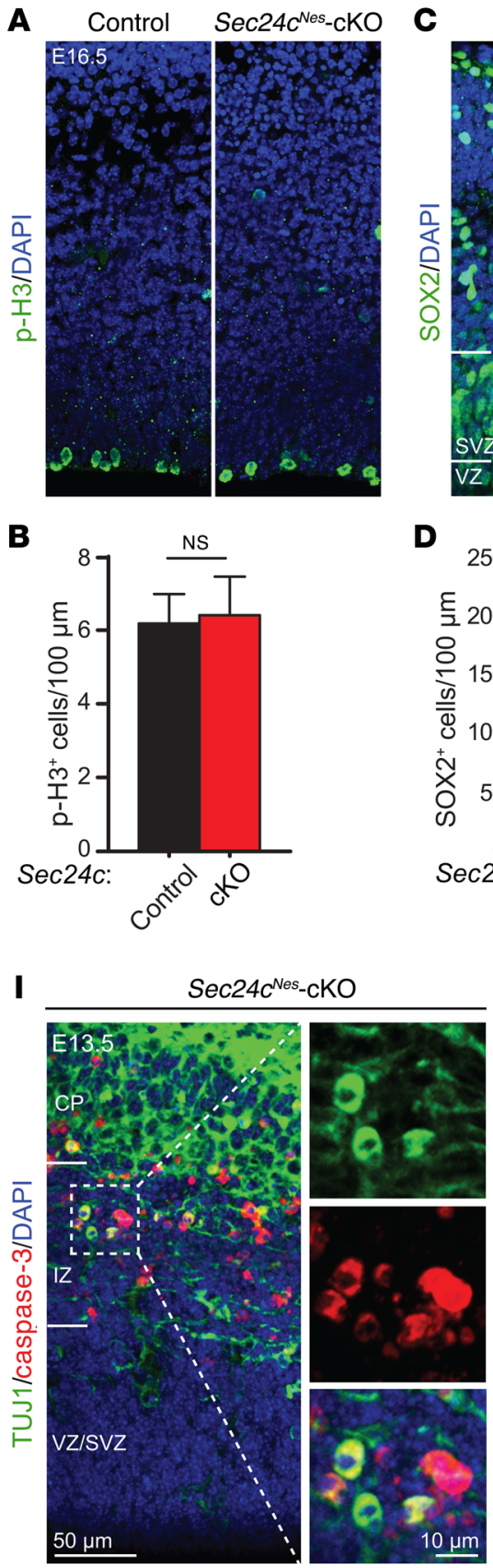
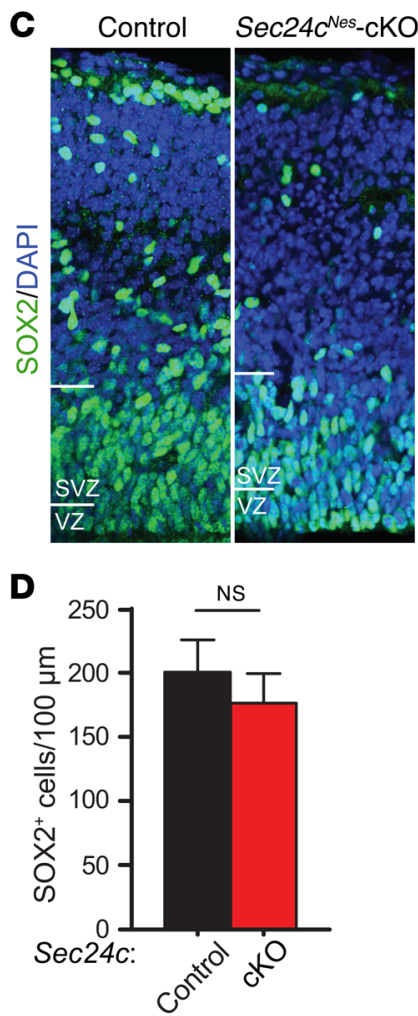
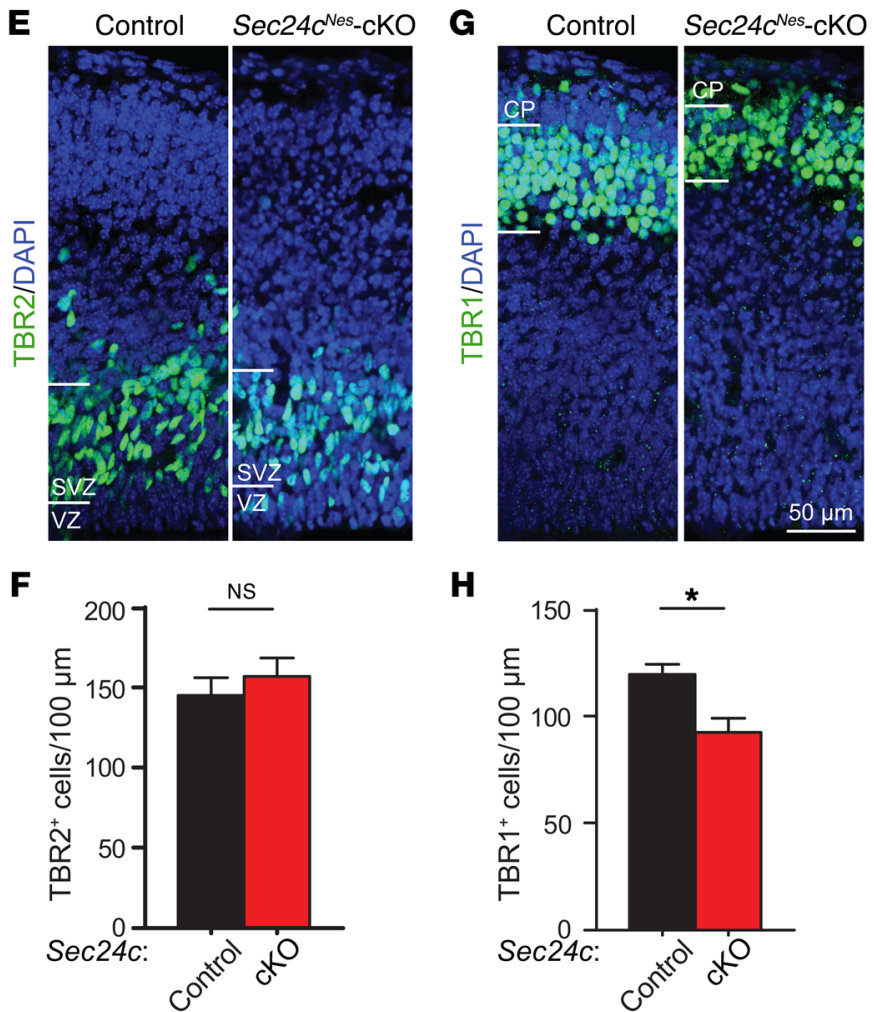

H

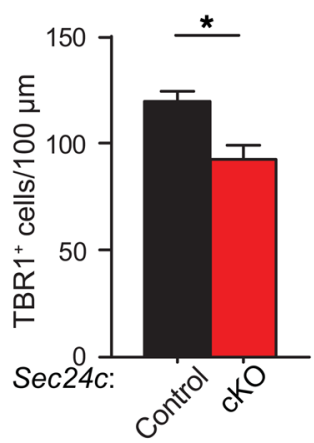

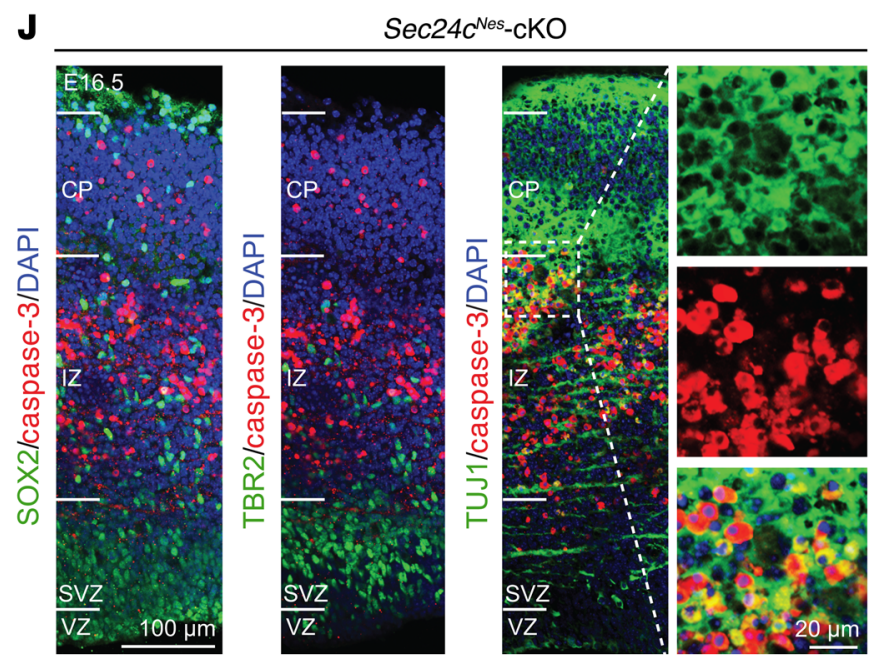

Figure 4. Sec24c deficiency triggers cell death in postmitotic neurons. (A-F) Representative images and mean number ( \pm SEM) of neurons in the VZ/SVZ region of the cortex from sections stained using antibodies against p-H3 (Ser10) (A and B), the neural stem cell marker SOX2 (C and $\mathbf{D})$, or the intermediate progenitor cell marker TBR2 (E and F) ( $n=3$ E16.5 mice per genotype). ( $\mathbf{G}$ and $\mathbf{H}$ ) Representative images of anti-TBR1-stained brain sections and mean number ( \pm SEM) of mature TBR $1^{+}$neurons (mean \pm SEM) in the cortical plate region ( $n=3$ E16.5 mice per genotype). Scale bar: $50 \mu \mathrm{m}$ (A, C, E, and G). (I) Representative image of a brain section from a E13.5 Sec24 $c^{\text {Nes }}$ - CKO mouse $(n=2)$ costained using antibodies against cleaved caspase-3 and the postmitotic neuronal cell marker TUJ1. Scale bars: $50 \mu \mathrm{m}$ and $10 \mu \mathrm{m}$ (insets). (J) Representative images of cortices from E16.5 Sec24 $c^{\mathrm{Nes}-c K O}$ mice $(n=2) \operatorname{costained}$ using antibodies against cleaved caspase-3 and SOX2, TBR2, or TUJ1. Scale bars: $100 \mu \mathrm{m}$ and $20 \mu \mathrm{m}$ (insets). ${ }^{*} P<0.05$, by Student's $t$ test.

the striatum persisted to birth (Figure 3, A and B). A TUNEL assay showed similar patterns of positivity in the brains of $\operatorname{Sec} 24 c^{\text {Nes }}$ - $\mathrm{KO}$ mice (Supplemental Figure 2). Together, these findings indicate that apoptosis contributes to the decreased number of neurons resulting from $\mathrm{Sec} 24 c$ deficiency.

Because defective neurogenesis caused by stem cell dysfunction may also lead to fewer neurons, we quantified cells at differ- ent stages of differentiation in the cortex of E16.5 mice. Neurogenesis begins in the ventricular and subventricular zones (VZ/SVZ) as $\mathrm{SOX}^{+}$neural stem cells transition into $\mathrm{TBR}^{+}$intermediate progenitors, which subsequently differentiate into mature TBR1 ${ }^{+}$ neurons as they migrate through the intermediate zone (IZ) to the cortical plate (CP) (36). Immunostaining for phosphorylated histone $\mathrm{H} 3$ (p-H3) highlights mitotic stem cells. We did not detect 
any difference in the number of $\mathrm{p}-\mathrm{H}^{+}$cells in the cortices of $\mathrm{Sec}$ $24 c^{\text {Nes }}$-cKO mice at E16.5 (Figure 4, A and B). Likewise, we found that the numbers of $\mathrm{SOX}^{+}$neural stem cells and TBR2 ${ }^{+}$intermediate progenitors were comparable (Figure $4, \mathrm{C}-\mathrm{F}$ ). In contrast, the abundance of $\mathrm{TBR} 1^{+}$neurons in the $\mathrm{CP}$ was significantly reduced in brains from $\mathrm{Sec} 24 c^{\text {Nes }}$ - $\mathrm{KO}$ mice compared with that in controls (Figure 4, G and $\mathrm{H}$ ). We detected activated caspase- 3 signal in the IZ and CP of E13.5 and E16.5 Sec $24 c^{\text {Nes }}$ - $\mathrm{CKO}$ mice, where it colocalized with TUJ1 (a marker for postmitotic neurons) but showed no overlap in staining with SOX2 or TBR2 (Figure 4, I and J). These results suggest that $\operatorname{Sec} 24 c$ deficiency in the embryonic brain causes preferential loss of mature postmitotic neurons in the CP.

Deletion of Sec24c leads to cell-autonomous degeneration of mature neurons. Nes-Cre is expressed in neurons and their supporting cells in the CNS during development, raising the possibility that the sensitivity of postmitotic neurons in $\operatorname{Sec} 24 c^{\text {Nes }}$ - $\mathrm{CKO}$ mice is secondary to the loss of $\operatorname{Sec} 24 \mathrm{c}$ expression in non-neuronal cells or due to developmental abnormalities. Therefore, we examined the consequences of deleting $\operatorname{Sec} 24 \mathrm{c}$ selectively from postnatal forebrain excitatory neurons by crossing $\operatorname{Sec} 24 c^{f / f l}$ mice with Camk2a-Cre-transgenic mice (37). By 12 months of age, the Sec $24 c^{f / / f t}$ Camk2a-Cre (hereafter referred to as $\operatorname{Sec} 24 c^{\text {Camk2a }}-\mathrm{cKO}$ ) mice showed a significant reduction in brain weight but not body weight compared with control mice (Sec24 $4 c^{f / f l}$ or Sec $24 c^{+/ f l}$ Camk2a-Cre) (Figure 5, A-C). Sec24c ${ }^{\text {Camk2a }}$-cKO mice had greater spontaneous locomotor activity in the open field test (Figure 5D), with no difference in time spent in the center of the open field (Figure 5E). The $\operatorname{Sec} 24 c^{\text {Camk2a }}$-cKO mice were also hyperactive in the elevated plus maze, spending significantly more time in the open arm (Figure $5 \mathrm{~F})$. Moreover, 2 of the 12-month-old Sec $24 c^{\text {Camk2a }}$-cKO mice tested jumped off the elevated maze; 1 of these mice jumped off 3 times during the 15-minute trial. None of the controls jumped off the maze, consistent with the rarity of such behavior in WT mice (38).

Nissl and Luxol fast blue staining of brain sections revealed a marked enlargement of the ventricles and thinning of cortices in 12-month-old Sec24c $c^{\text {Camk2a }}$-cKO mice (Figure 5, G and $\mathrm{H})$. Immunostaining for the neuronal marker NeuN showed a significant decrease in the number of mature neurons in the cortices of 12-month-old Sec $24 c^{\text {Camk2a }}$-cKO mice compared with numbers in the control mice (Figure 5, G and I). We observed no obvious abnormalities in the anatomical structures of the forebrain in 2-month-old Sec24 $4 c^{\text {Camk2a }}$-cKO mice (Supplemental Figure 3A). These findings indicate that $\operatorname{Sec} 24 \mathrm{c}$ deficiency causes a progressive cell-autonomous loss of mature neurons. According to the data from the Allen Brain Atlas (http://www. brain-map.org/) (39), the level of Sec24c expression is normally higher than that of the other Sec24 paralogs in the adult mouse cortex (Supplemental Figure 3B).

$S e c 24 c$ deficiency induces the unfolded protein response and $E R$ stress. Perturbations in ER-to-Golgi trafficking can lead to the retention of excessive amounts of protein in the ER and subsequent induction of the unfolded protein response (UPR) (40). The UPR is mediated by 3 ER-localized protein sensors (i.e., ATF6, IRE1, and PERK), which, upon activation, trigger a series of responses to counterbalance protein accumulation (41). To determine whether neuronal cell death resulting from $\operatorname{Sec} 24 \mathrm{c}$ deficiency was associated with UPR activation, we analyzed the levels of representative UPR genes by real-time RT-PCR. We isolated RNA from the cortices of E13.5 Sec $24 c^{\text {Nes }}$-cKO embryos and controls, because the loss of postmitotic neurons is more synchronous in $\operatorname{Sec} 24 c^{\text {Nes }}$ - $\mathrm{CKO}$ embryos than in $\operatorname{Sec} 24 c^{\mathrm{Camk2a}}$-cKO adults. The UPR genes Atf4, Chop, Gadd34, Herp, BiP, and sXbp1 were upregulated in the cortices of $\operatorname{Sec} 24 c^{\text {Nes }}$-cKO mice, indicating the activation of all 3 major UPR pathways (Supplemental Figure 4). Despite the increased levels of $s X b p 1$, the expression of the 2 XBP1-specific targets, Edem1 and ERdj4, which regulate ER-associated degradation (42-44), remained unchanged (Supplemental Figure 4).

The prolonged activation of the UPR and failure to alleviate ER stress can activate intrinsic and extrinsic apoptotic pathways. C/EBP homologous protein (CHOP) is a key mediator of ER stress-induced apoptosis (40). Given the increase in Chop mRNA in the brains of $\mathrm{Sec} 24 \mathrm{C}^{\text {Nes }}$-cKO mice (Supplemental Figure 4), we examined the levels and subcellular distribution of CHOP in different brain regions. CHOP levels and nuclear localization were increased in striatal neurons of E13.5 Sec $24 c^{\text {Nes }}$-cKO mice (Figure 6A). Nuclear translocation of CHOP was also evident in the cortices of E13.5 Sec $24 \mathrm{c}^{\text {Nes }}$ - $\mathrm{CKO}$ mice, but only in cells located at the upper CP (Figure 6B). Transmission electron microscopy (EM) of the striatum of $\mathrm{Sec} 24 \mathrm{c}$-deficient mice revealed cells with a swollen, dilated ER, unlike the thin, elongated ER decorated with numerous ribosomes in the cells of control striatum (Figure 6C). E13.5 cortical neurons were examined using both scanning and transmission EM, with the former allowing orientation of neurons within the cortex and the latter providing superior resolution. These ultrastructural studies confirmed the swollen ER in cells in the CP but not the VZ/SVZ of Sec $24 c^{\text {Nes }}$ - CKO mice (Figure 6D). By E16.5, the cortices of $\operatorname{Sec} 24 c^{\text {Nes }}$-cKO mice showed prominent nuclear localization of CHOP in cells in the IZ (Figure 6E). The reduced number of mature $\mathrm{TBR} 1^{+}$neurons in the $\mathrm{CP}$, the increase in cleaved caspase- 3 positivity, and the increase in nuclear $\mathrm{CHOP}$ in neurons within the IZ indicated that postmitotic neurons are particularly sensitive to $S e c 24 c$ deficiency.

Because Chop inactivation delays cell death in murine models of several diseases, including Parkinson's disease (40, 45), we tested its impact on postmitotic neurons in $\operatorname{Sec} 24 c^{\text {Nes }}$ - $\mathrm{CKO}$ mice. We interbred Sec $24 c^{\text {Nes }}$-cKO mice with Chop-KO $\left(\mathrm{Chop}^{-/-}\right)$mice and examined the phenotypes of Sec2 $4 c^{\text {Nes }}$-cKO Chop-KO $\left(\operatorname{Sec} 24 c^{f / f l}\right.$ Nes-Cre Chop $\left.{ }^{-/-}\right)$mice and their littermate controls $\left(\mathrm{Sec} 24 \mathrm{c}^{+/ f l} \mathrm{Nes}-\right.$

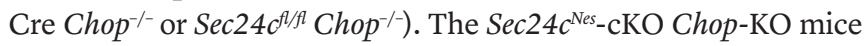
showed microcephaly, with brain weights significantly lower than those of their littermate controls (Supplemental Figure 5, A and B) but comparable to those of $\operatorname{Sec} 24 c^{N e s}$ - $\mathrm{CKO}$ mice (Figure 1, B and C).

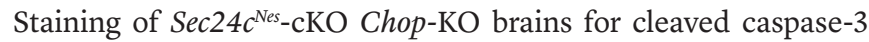
showed widespread cell death (Supplemental Figure 5C), similar to that observed in Sec24 $4 c^{\text {Nes }}$-cKO mice (Figure 3A). At E16.5, cortical neurons at different stages of differentiation showed no differences in the numbers of $\mathrm{SOX}_{2}^{+}$neural stem cells or $\mathrm{TBR} 2^{+}$intermediate progenitors between $\mathrm{Sec} 24 \mathrm{c}^{\mathrm{Nes}}$-cKO Chop-KO mice and their littermate controls (Supplemental Figure 5, D and E). As with Sec-

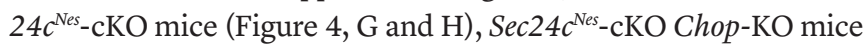
showed a significant loss of mature TBR $1^{+}$neurons compared with that detected in their littermate controls (Supplemental Figure 5F). The inactivation of Chop failed to delay the death of Sec24c-deficient neurons, suggesting that 1 or more pathways mediating ER 
A

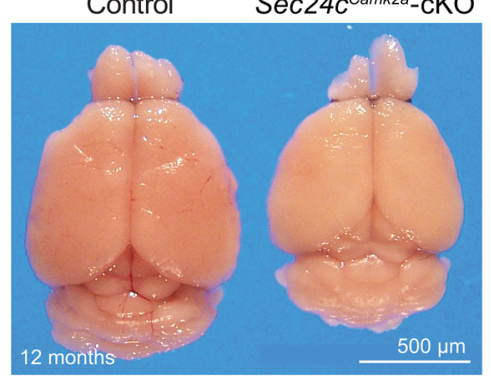

B

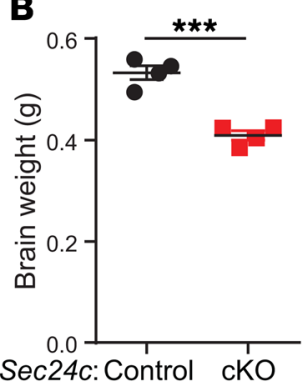

$\mathbf{F}$

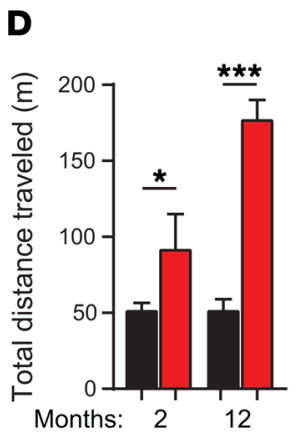

$\mathbf{E}$

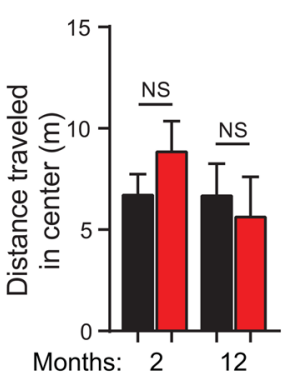

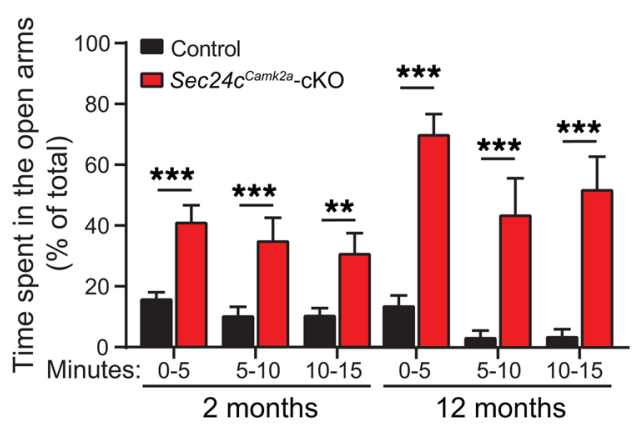

G
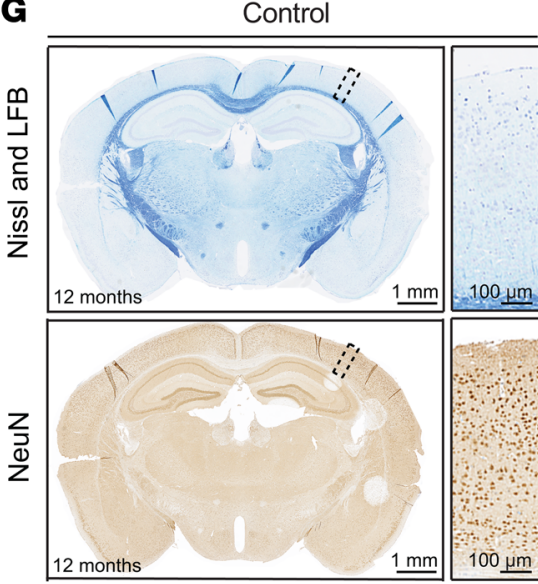

C

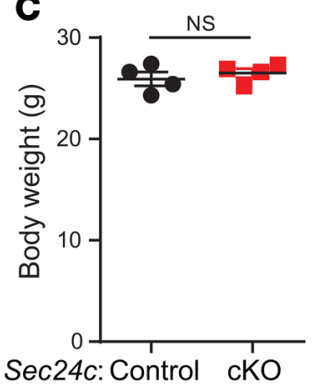

12 months
Sec24c ${ }^{\text {Camk2a }}$-cKO

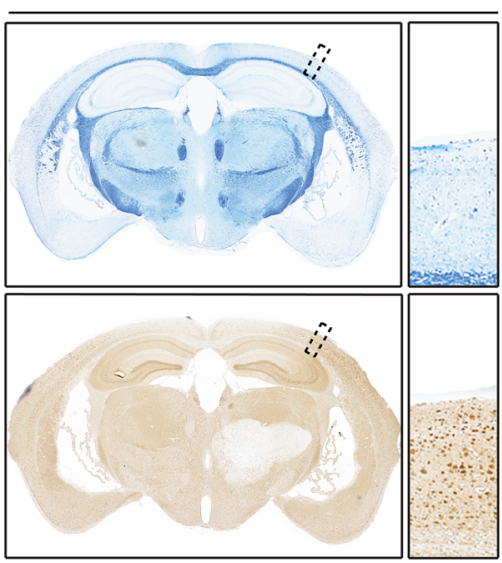

H

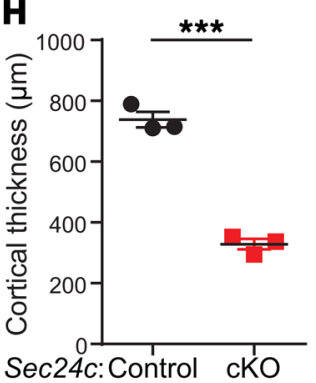

I

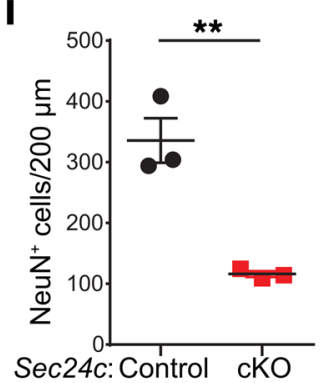

Figure 5. Postnatal deletion of Sec24c in the forebrain leads to hyperactivity and altered anatomy in the forebrain. (A) Representative image of fixed brains dissected from a 12-month-old Sec24c camk2a-cKO mouse and littermate control. Scale bar: $500 \mu \mathrm{m}$. (B and C) Brain (B) and body (C) weights (mean \pm SEM) of 12-month-old Sec24c $c^{\text {Camk2a }}$-cKO and control mice $(n=4$ mice/genotype). (D-F) Behavioral tests were performed using 2-month-old Sec24c ${ }^{\text {Camk2a }}$-cKO mice $(n=8)$ and agematched controls $(n=17)$ and 12-monthold Sec24c camk2a - cKO mice $(n=4)$ and agematched controls $(n=5)$. Total distance traveled (mean \pm SEM) (D) and distance traveled in the center (mean \pm SEM) (E) by mice subjected to the open field test. (F) Mean time ( \pm SEM) spent in the open arms during the indicated intervals by mice subjected to the elevated plus maze test. (G-I) Brain sections from 12-monthold Sec24c ${ }^{\text {Camk2a }}$-cKO and littermate control mice ( $n=3$ mice/genotype) were stained with Nissl and Luxol fast blue or immunostained with anti-NeuN antibody. Representative images (G), mean cortical thickness ( \pm SEM) $(\boldsymbol{H})$, and mean neuronal numbers ( \pm SEM) (I) are shown. (G) Scale bars: $1 \mathrm{~mm}$ and $100 \mu \mathrm{m}$ (insets). LFB, Luxol fast blue. ${ }^{*} P<0.05,{ }^{* *} P<0.01$, and ${ }^{* *} P$ $<0.001$, by Student's $t$ test. stress-induced cell death compensates for the loss of CHOP or plays a more prominent role than $\mathrm{CHOP}$ in this process.

Deletion of SEC24C leads to reduced viability of differentiated neurons in culture. Given the complex relationships between neurons and supporting cells in the brains of $\operatorname{Sec} 24 c^{\text {Nes- }}$-cKO mice, we sought to examine the impact of SEC24C deficiency on the differentiation and viability of neurons in culture. Toward this end, we used CRISPR-Cas9 technology to target SEC24C expression in human induced pluripotent stem cells (hiPSCs) and then used established protocols to differentiate those cells into neurons (46). Immunoblot analyses confirmed loss of SEC24C in the $2 \mathrm{KO}$ hiPSC lines (clones 1 and 2) (Figure 7A). The levels of SEC24D appeared mildly elevated in the SEC24C-KO hiPSC lines, whereas SEC24A and SEC24B levels remained unchanged (Figure 7A). The percentage of differentiated cells stained by TUJ1 did not significantly differ between the WT and SEC24C-KO lines (Fig- 
A
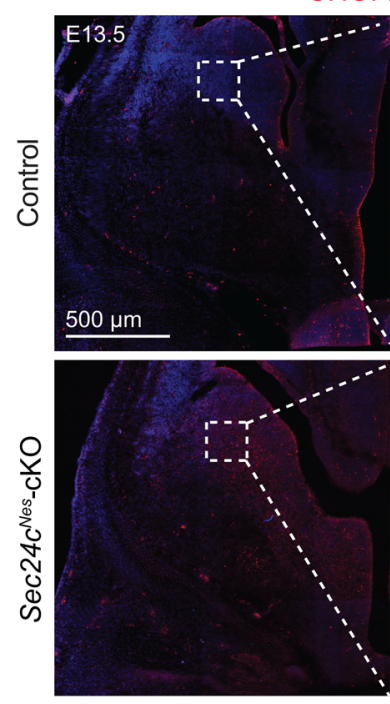

D
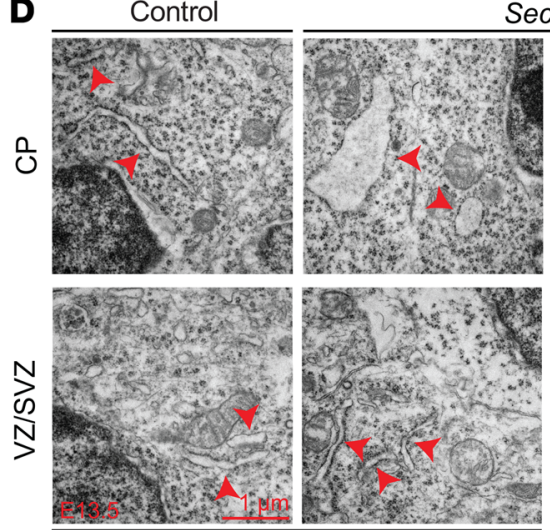

Transmission EM

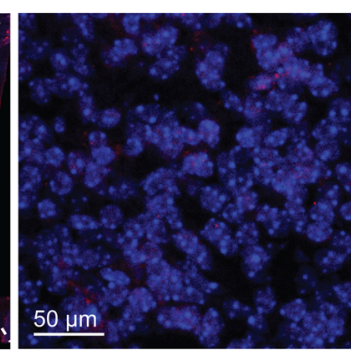

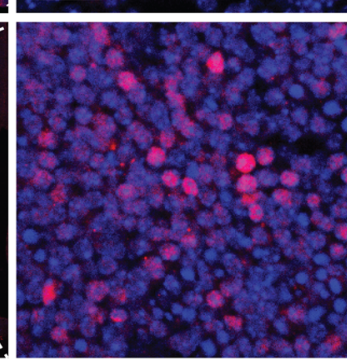

B

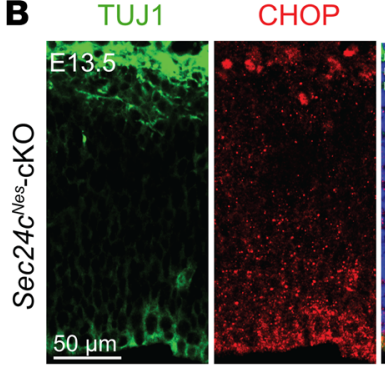

C Control

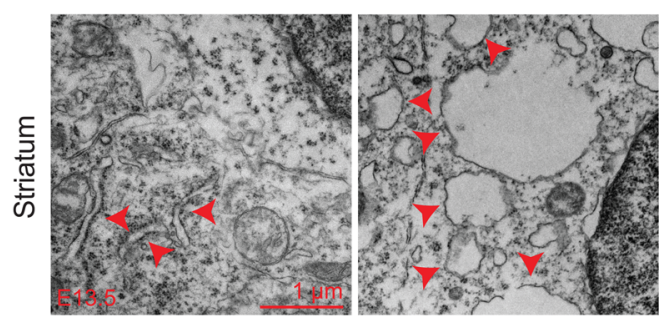

Merge

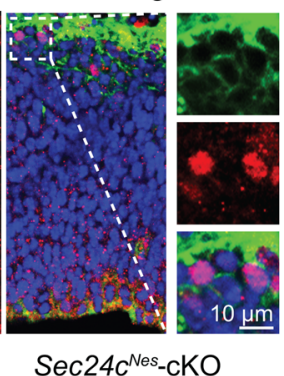

$\operatorname{Sec} 24 c^{\text {Nes-cKO }}$

\section{Pial}

\section{E}

TUJ1/CHOP/DAPI

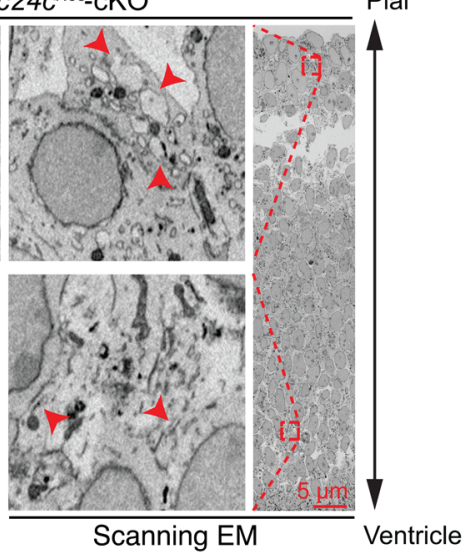

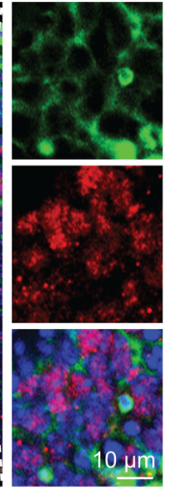

Figure 6. Sec24c deficiency causes ER stress. (A) Representative images of immunostaining against CHOP in the striatum of control ( $n=3$ ) and Sec$24 c^{\text {Nes }}$-cKO $(n=3)$ mice at E13.5. Scale bars: $500 \mu \mathrm{m}$ and $50 \mu \mathrm{m}$ (insets). (B) Representative images of CHOP staining in the cortex of Sec24c $c^{\text {Nes }}$ - CKO ( $n=3$ ) mice at E13.5. Scale bars: $50 \mu \mathrm{m}$ and $10 \mu \mathrm{m}$ (insets). (C) Representative ultrastructural images of E13.5 striatal neurons showing a swollen ER (denoted by red arrowheads) in Sec24c-deficient brain $(n=1)$. Scale bar: $1 \mu \mathrm{m}$. (D) Scanning and transmission EM analyses of the ultrastructure in cells located in the CP and the VZ/SVZ in Sec24 Nes -cKO mice $(n=1)$. The ER structure is highlighted by arrowheads. Scale bars: $1 \mu \mathrm{m}$ and $5 \mu \mathrm{m}$ (inset). (E) Representative images of CHOP staining in the cortex of Sec $24 c^{\text {Nes }}$-cKO mice at E16.5 $(n=3)$. Scale bars: $50 \mu \mathrm{m}$ and $10 \mu \mathrm{m}$ (insets).

ure 7, B and C), suggesting that disruption of SEC24C expression did not inhibit neuronal differentiation. Nevertheless, the levels of CHOP mRNA, which increased in both WT and SEC24C-KO lines during the differentiation process, were significantly higher in neurons derived from both SEC24C-KO hiPSC lines compared with levels in the unedited WT cells (Figure 7D). Moreover, immunostaining revealed increased levels of CHOP in the nuclei of neurons derived from SEC24C-KO hiPSCs compared with those derived from WT cells (Figure 7, E and F). Immunofluorescence microscopy also showed significantly higher proportions of cells with condensed $\mathrm{DAPI}^{+}$nuclei in the SEC24C-KO hiPSC-derived neuron cultures compared with cells in WT cultures (Figure 7, G and H), suggesting reduced cell viability (47). We also detected a significant decrease in the level of cellular dehydrogenase enzymes in SEC24C-KO hiPSC-derived neurons by XTT cell viability assay (Roche) during the final weeks of cul- ture, consistent with a differentiation-induced decrease in the viability of the cells (Supplemental Figure 6). Collectively, our data suggest that SEC24C plays an important homeostatic role in neurons in vivo and in vitro.

$S E C 24 D$ rescues the neuronal loss resulting from Sec $24 c$ deficiency. The sensitivity of neurons to the loss of SEC24C was surprising, given that $\operatorname{Sec} 24 c$ deficiency is well tolerated by other professional secretory cells (32). Nevertheless, this finding suggested that SEC24C has a unique function in neurons, either because of its expression pattern or cargo specificity. ISH studies from the Genepaint database (https://gp3.mpg.de/) (48) demonstrated that Sec24c is widely expressed throughout the cortex at E14.5 but that Sec24d transcripts are highest in the VZ/SVZ (i.e., regions enriched in stem/progenitor cells) (Supplemental Figure 7A). Review of RNA-sequencing data from the DeCoN database (http://decon.fas.harvard.edu/pyrami$\mathrm{dal} /$ ) (49) suggested that $\operatorname{Sec} 24 \mathrm{c}$ is expressed at higher levels than 
A

\begin{tabular}{|c|c|c|c|c|c|}
\hline \multicolumn{6}{|c|}{ Differentiation (weeks) } \\
\hline & 0 & & 3 & & 6 \\
\hline 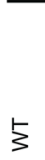 & 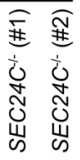 & 々 & 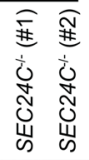 & 5 & 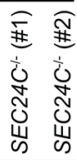 \\
\hline- & & & & & \\
\hline
\end{tabular}

$\begin{array}{llllllllll}\text { SEC24C/GAPDH: } & 1.0 & 0.1 & 0.1 & 6.4 & 1.4 & 1.1 & 6.7 & 0.7 & 1.1\end{array}$

SEC24D

SEC24D/GAPDH: $1.0 \quad 4.7 \quad 7.0 \quad 4.5 \quad 6.5 \quad 4.1 \quad 3.2 \quad 8.7 \quad 9.5$ SEC24A

$\begin{array}{llllllllll}\text { SEC24A/GAPDH: } & 1.0 & 1.1 & 1.1 & 1.0 & 1.0 & 1.0 & 0.7 & 0.6 & 0.4\end{array}$

SEC24B

SEC24B/GAPDH: $\begin{array}{llllllllll}1.0 & 0.7 & 0.7 & 1.0 & 0.6 & 0.6 & 0.8 & 0.8 & 0.4\end{array}$

GAPDH

D
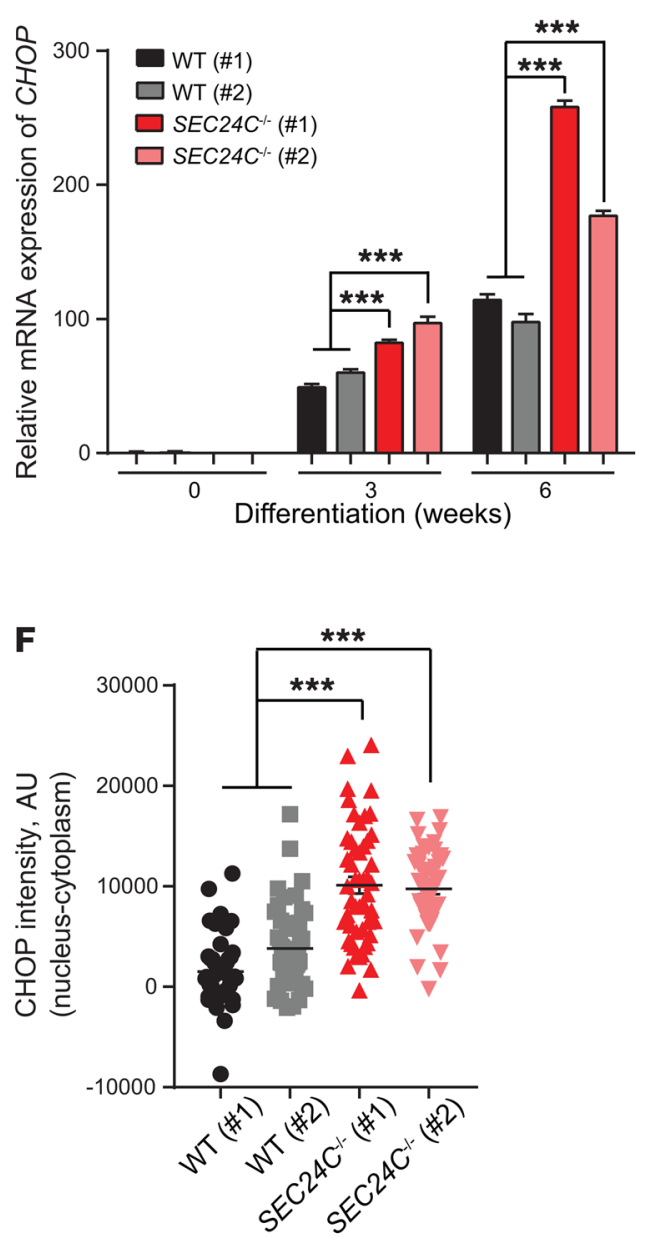

B
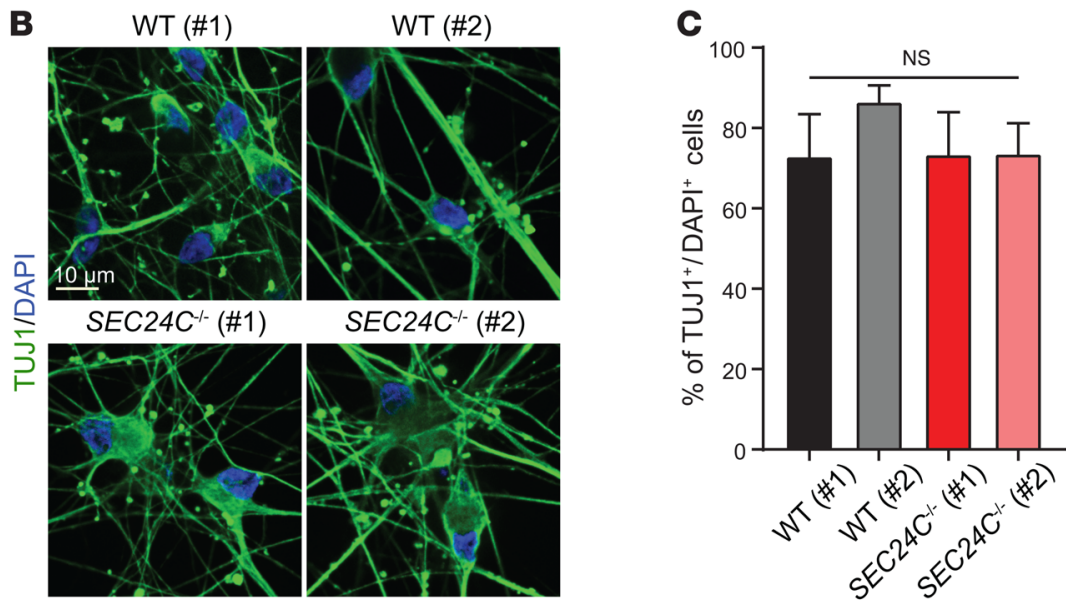

E

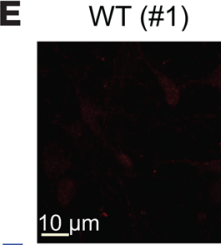

WT (\#2)

SEC24C-1- $(\# 1)$

$S E C 24 C^{-1-}(\# 2)$
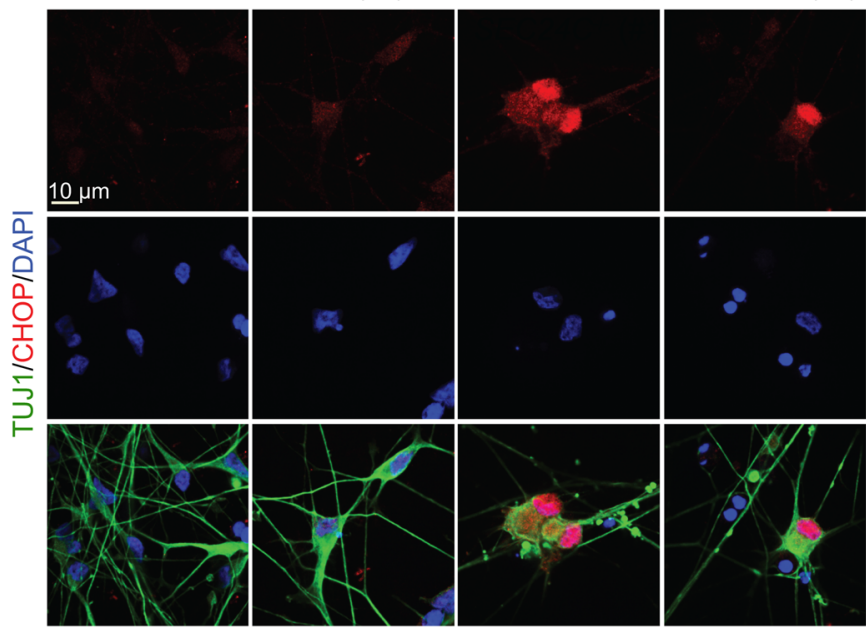

G

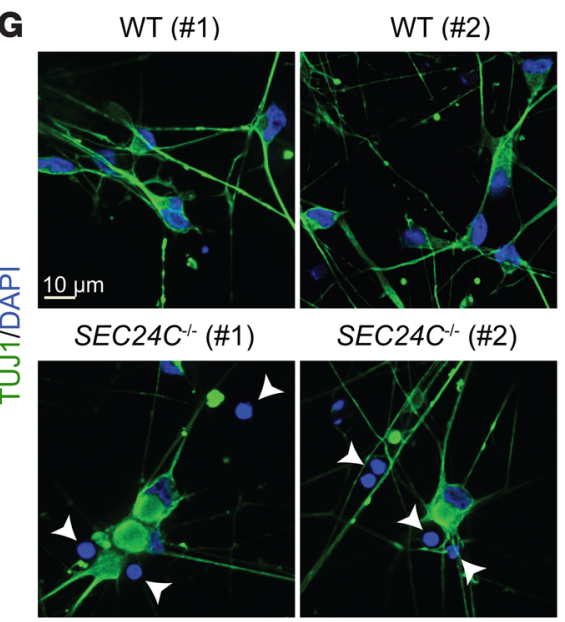

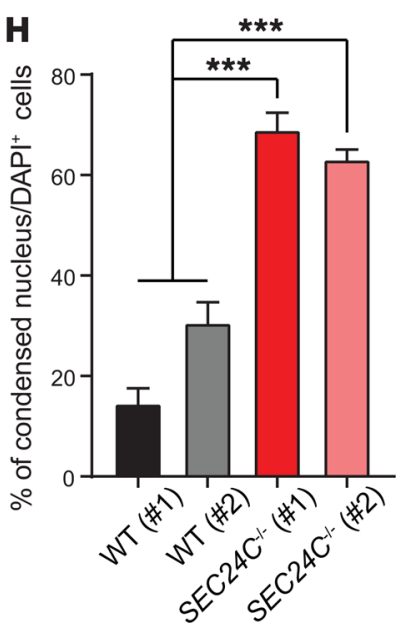

Figure 7. Deletion of SEC24C leads to elevated cellular stress and the demise of mature neurons derived from hiPSCs. (A) Immunoblot analysis of cell extracts from WT and SEC24C-KO hiPSCs (clones 1 and 2) at 0, 3, and 6 weeks of neuronal differentiation. (B) Representative images of TUJ1 immunostaining of WT and SEC24C-KO hiPSCs at 6 weeks of differentiation. Scale bar: $10 \mu \mathrm{m}$. (C) Percentage of TUJ1 $1^{+}$cells per total cells (DAPI+) (mean \pm SEM) shows normal differentiation of the SEC24C-KO hiPSCs compared with WT clones 1 and 2. (D) mRNA levels of CHOP in WT and SEC24C-KO hiPSCS at 0, 3, and 6 weeks of differentiation were determined by real-time RT-PCR. Data are presented as the mean \pm SEM. (E) Representative images of immunostaining show pronounced nuclear localization of CHOP in SEC24C-KO hiPSCs at 6 weeks of differentiation. Scale bar: $10 \mu \mathrm{m}$. (F) Quantification of nucleus minus cytoplasm CHOP intensity shows significant enrichment of nuclear CHOP in SEC24C-KO hiPSCs. (C) Representative nuclear staining with DAPI and (H) quantification indicate a significant increase in the percentage of cells with condensed nuclei (mean \pm SEM) in SEC24C-KO hiPSCs. Scale bar: $10 \mu \mathrm{m}$. Data were collected from 3 independent experiments. ${ }^{* *} P<0.001$, by 2 -way ANOVA. 
its paralogs Sec24a, Sec24b, and Sec24d in the callosal (layers II-III) and subcerebral (layer V) neurons of WT mice at all developmental stages examined (Supplemental Figure 7, B and C). As mentioned previously, data from the Allen Brain Atlas (48) showed that the level of $\operatorname{Sec} 24 c$ expression is higher than that of the other Sec24 paralogs in the cortex of adult WT mice (Supplemental Figure 3B). We also compared the levels of $\operatorname{Sec} 24 \mathrm{cmRNA}$ and protein in the brains of WT mice at different developmental stages (i.e., E13.5, E16.5, P0, P7, P21, and P56). We found that Sec $24 c$ mRNA expression declined during development, whereas protein expression peaked at PO (Supplemental Figure 7, D and E), coinciding with the peak of neurogenesis in the developing cortex. Together, these data suggest that SEC24C is the major cargo adapter for COPII-dependent transport in postmitotic neurons in developing and adult brains, which may help to explain the sensitivity of these cells to loss of SEC24C.

To explore the extent of functional overlap between SEC24C and SEC24D in the brain, we used a recently developed knockin mouse model, in which $\operatorname{Sec} 24 \mathrm{c}$ exons were replaced by corresponding sequences from $\operatorname{Sec} 24 d\left(\operatorname{Sec} 24 c^{c-d}\right)$, resulting in a $\operatorname{Sec} 24 c-d$ fusion gene (34). Immunoblot analyses confirmed the loss of SEC24C expression and the increase in SEC24D expression in the brains of $\operatorname{Sec} 24 c^{c-d / c-d}$ mice (Figure 8A). The embryonic lethality at E7.5, which was associated with germline disruption of Sec $24 c$ expression, was rescued by the expression of the SEC24C-SEC24D fusion protein in $\operatorname{Sec} 24 c^{c-d / c-d}$ mice. However, these mice failed to suckle and died shortly after birth. Although the brains of E16.5 Sec24 $4 c^{c-d / c-d}$ mice showed minimal reductions in size and weight compared with those measures in littermate controls ( $\operatorname{Sec} 24 c^{+/+}$or $\operatorname{Sec} 24 c^{+/ c-d}$ ) (Figure 8, B and $\mathrm{C})$, the ratio of brain weight to body weight in $\operatorname{Sec} 24 c^{c-d / c-d}$ mice was marginally increased relative to that of littermate controls (Figure $8 D)$, because their overall body size and weight were reduced (34). Moreover, unlike the brains of E16.5 Sec $24 c^{\text {Nes }}$-cKO mice, the brains of $\operatorname{Sec} 24 c^{c-d} / c-d$ mice showed no increase in cleaved caspase- 3 staining compared with that in littermate controls (Figure 8, E and F). Finally, we detected no difference in the numbers of $\mathrm{SOX} 2^{+}$neural stem cells, $\mathrm{TBR}^{+}$neural progenitor cells, or $\mathrm{TBR} 1^{+}$postmitotic cells in the brains of $\operatorname{Sec} 24 c^{c-d / c-d}$ mice or those of their littermate controls (Figure $8, \mathrm{G}$ and $\mathrm{H}$ ). These results demonstrate that ectopic expression of $\operatorname{Sec} 24 d$ is sufficient to prevent the loss of neurons resulting from $\operatorname{Sec} 24 c$ deficiency in the brains of developing mice.

\section{Discussion}

Our results demonstrate that postmitotic neurons in developing and adult mouse brains are uniquely sensitive to loss of the COPII cargo adapter SEC24C. Given the expression pattern of the Sec24 isoforms in the brains of developing mice, we speculate that sensitivity of these cells to loss of $\operatorname{Sec} 24 \mathrm{C}$ may be related to the decreased levels of Sec24d in neurons transitioning out of the VZ/SVZ, which may not be sufficient to support the demand for SEC24C-SEC24D-dependent ER export in the absence of Sec24c expression. Indeed, the normal brain architecture (i.e., size, cortical cell numbers, and absence of cleaved caspase-3 positivity) in $\operatorname{Sec} 24 c^{c-d / c-d}$ embryos in which SEC24C expression was replaced by SEC24D suggests that SEC24D can compensate for the loss of SEC24C during development. However, the death of $\operatorname{Sec} 24 c^{c-d / c-d}$ mice in the immediate postnatal period suggests that there may be specific cargo in the brain or other tissues of $\operatorname{Sec} 24 \mathrm{c}$-deficient animals for which the defect in ER-to-Golgi trafficking cannot be overcome by ectopic expression of SEC24D. Additional studies are required to determine whether the Sec24c- $d$ allele can rescue ER-to-Golgi trafficking of SEC24C-specific cargo and/or behavioral defects resulting from loss of SEC24C in postmitotic neurons postnatally (i.e., in $\operatorname{Sec} 24 c^{\text {Camk2a }}$-cKO mice), especially since mice lacking SEC24C-specific cargo, such as SERT (also known as SLC6A4), exhibit elevated baseline anxiety (50).

Postmitotic neurons express higher levels of SEC24C than do other SEC24 isoforms and are clearly sensitive to the loss of SEC24C, but it remains to be seen how neuronal function is affected by the loss of the other SEC24 isoforms. Although Sec24a-KO mice do not show any obvious neurodevelopmental abnormalities (32), the severe defect in neural tube closure in Sec24b-KO mice and early embryonic lethality of Sec $24 d$-KO mice (33) preclude the use of these germline deletion models to assess the role of SEC24B and SEC24D during neuronal differentiation (27). Follow-up studies examining the role of SEC24C and other SEC24 paralogs in different neuronal subtypes, particularly in adult animals, may reveal additional neuronal vulnerabilities due to defects in ER-to-Golgi trafficking of specific cargoes.

Consistent with its role in ER-to-Golgi trafficking, SEC24C deficiency in postmitotic neurons (in vivo and derived from hiPSCs) was associated with ER stress and activation of the UPR. Susceptible cells in mice lacking other COPII proteins such as SEC23A or SEC23B also showed ER stress and UPR activation, particularly of the PERK/ eIF2 $\alpha / \operatorname{ATF} 4 / C H O P$ pathway $(13,14)$. As the upregulation and nuclear localization of CHOP has been observed in many COPII-defective models, we examined the impact of CHOP inactivation on neuronal viability in $\operatorname{Sec} 24 c^{\text {Nes }}$-cKO mice. Although Chop inactivation can delay cell death in numerous disease models, such as that for Parkinson's disease $(40,45)$, abolishing Chop alone in Sec $24 c^{\text {Nes }}$-cKO mice did not alter the induction of apoptotic death in postmitotic neurons. Thus, the benefit of abolishing CHOP function in the context of pathologic conditions may depend on the extent and duration of ER stress induction. Under extreme conditions, wherein all arms of the UPR are activated, targeting CHOP alone might not be sufficient to reverse cell death. Although several drugs targeting $\mathrm{CHOP}$ are being explored to treat human diseases associated with ER stress $(51,52)$, strategies that target multiple branches of UPR pathways may be required to effectively circumvent the chronic ER stress associated with certain pathologic conditions.

\section{Methods}

Animals. Sec24ct/fl (stock 024867), Nes-Cre (stock 003771), Camk2a-Cre (stock 005359), and Chop ${ }^{-/}$(stock 005530) mice were obtained from The Jackson Laboratory. $\operatorname{Sec} 24^{c-d}$ mice were generated as described previously (34). Briefly, a targeting construct containing the coding sequence of SEC24D, spanning from Val41 to Asn1032, was microinjected into $\operatorname{Sec} 24 c^{G T}$ embryonic stem cells (32) to replace exons 3-23 of SEC24C through dual recombinase-mediated cassette exchange. The targeted mice express a SEC24C-SEC24D fusion protein, which consists of the first 57 amino acids of SEC24C followed by the SEC24D coding sequence and a stop codon. All mice were maintained on a C57BL/6 background. Both male and female mice were included in our analyses. 
A

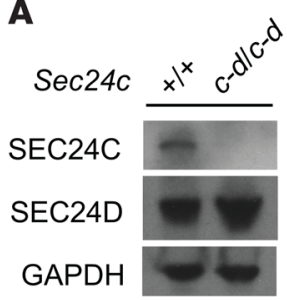

E

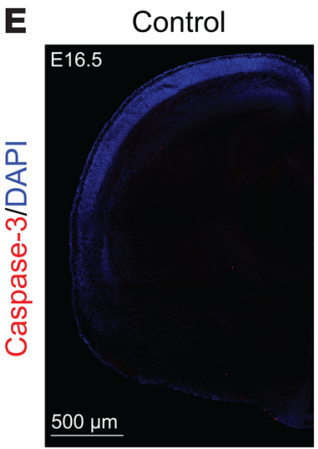

B

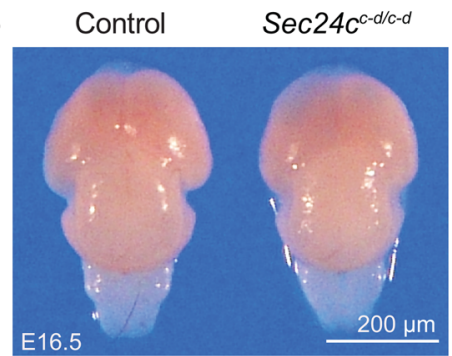

$\operatorname{Sec} 24 C^{c-d / c-d}$

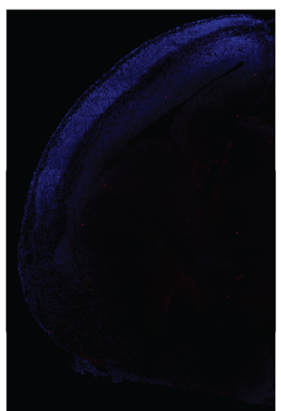

$\operatorname{Sec} 24 C^{\text {Nes-cKO }}$

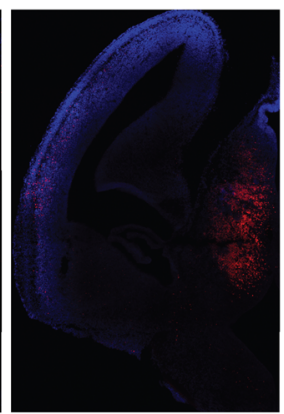

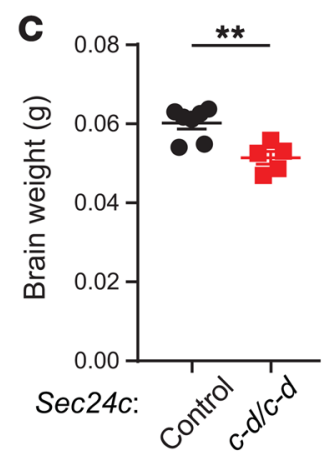

$\mathbf{F}$

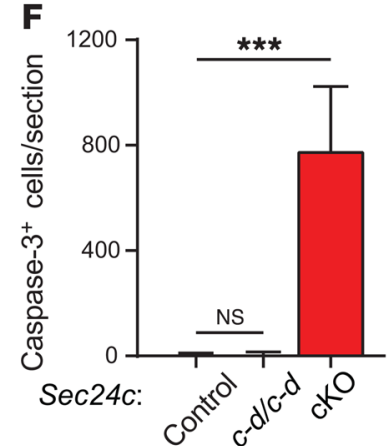

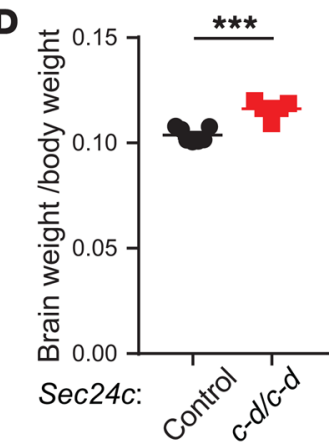
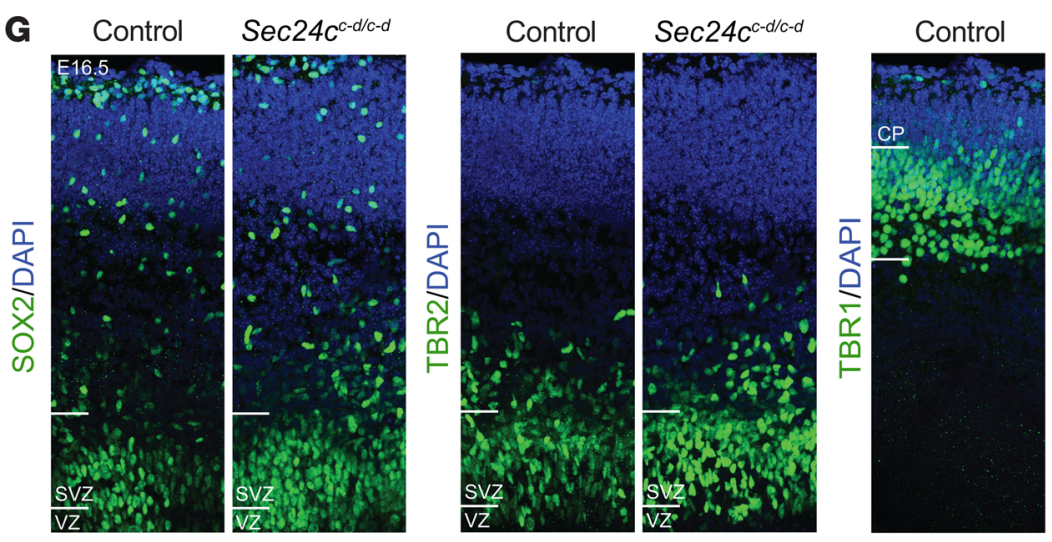

$\operatorname{Sec} 24 c^{c-d / c-d}$

H

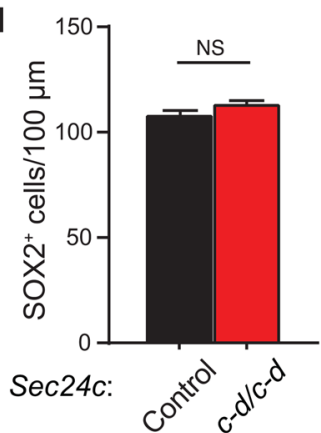

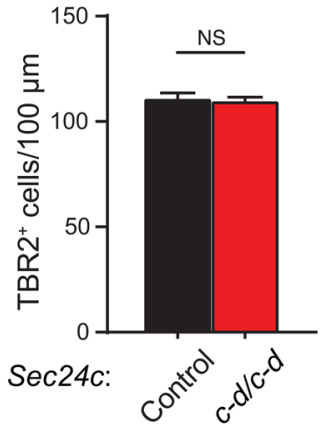

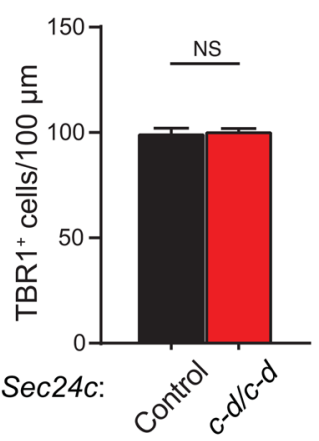

Figure 8. SEC24D can replace SEC24C during development of the embryonic mouse brain. (A) Western blot of brain extract prepared from Sec24 $c^{+/+}(n=1)$ and Sec24c $c^{c-d / c-d}$ mice $(n=1)$ shows almost complete abolishment of SEC24C and elevated expression of SEC24D. (B and C) Representative photograph (B) and mean $\left( \pm\right.$ SEM) fixed brain weight $(\mathbf{C})$ of control $(n=7)$ and $\operatorname{Sec} 24 c^{c-d / c-d}(n=5)$ mice at E16.5. Scale bar: $200 \mu$ m. (D) Average ratio $( \pm$ SEM) of brain weight to body weight in $\operatorname{Sec} 24 c^{c-d / c-d}$ mice $(n=5)$ compared with that of control mice $(n=7)$. (E and $\left.\mathbf{F}\right)$ Representative images of cleaved caspase-3 staining $(\mathbf{E})$ and mean number $\left( \pm\right.$ SEM) of cleaved caspase- $3^{+}$cells revealed no obvious cell death in the brains of Sec $24 c^{c-d / c-d}$ mice. Brain sections from Sec $24 c^{\text {Nes }-c K O}$ mice at the same age were used as a positive control ( $n=3$ mice/genotype). Scale bar: $500 \mu \mathrm{m}$. ( $\mathbf{C}$ and $\mathbf{H})$ Representative images and the number of SOX2+, TBR2 $2^{+}$, and TBR $1^{+}$cells (mean \pm SEM) were normal in the Sec $24 c^{c-d / c-d}$ mouse brain $(n=3)$ compared with control mice $(n=3)$. Scale bar: $50 \mu \mathrm{m}$. ${ }^{* *} P<0.01$ and ${ }^{* *} P<0.001$, by Student's $t$ test. 
Immunostaining and histology. Brains from embryonic and newborn mice were dissected in PBS and fixed overnight in $4 \%$ paraformaldehyde (PFA) in PBS at $4^{\circ} \mathrm{C}$. The brains were then cryoprotected using $30 \%$ sucrose in PBS overnight at $4^{\circ} \mathrm{C}$ and embedded in OCT compound for cryosectioning. Next, 20- $\mu \mathrm{m}$ frozen sections were washed with $0.2 \%$ Triton X-100 in Tris-buffered saline (TBST) and incubated in blocking solution (5\% normal goat serum in TBST) for 1 hour at room temperature. Sections were incubated with primary antibodies diluted in the blocking solution overnight at $4^{\circ} \mathrm{C}$, washed with TBST, and incubated with Alexa Fluor-conjugated secondary antibodies (Invitrogen, Thermo Fisher Scientific) diluted at 1:1,000 in the blocking solution for 2 hours at room temperature. Sections were mounted in ProLong Gold Antifade Reagent with DAPI (Invitrogen). The following primary antibodies were used: mouse anti-SATB2 (Abcam, ab51502); rat anti-CTIP2 (Abcam, ab18465); rabbit anti-TBR1 (Abcam, ab31940); rabbit antiTBR2 (eBioscience, 14-4875-82); mouse anti-ISLET1/2 (Developmental Studies Hybridoma Bank, 39.4D5); rabbit anti-NKX2.1 (Abcam, ab76013); rabbit anti-cleaved caspase-3 (Cell Signaling Technology, 9661); mouse anti-TUJ1 (Covance, MMS-435P); goat anti-SOX2 (Santa Cruz Biotechnology, TX; sc-17320); rabbit antip-H3 (MilliporeSigma, 06-570); and rabbit anti-CHOP (Santa Cruz Biotechnology, sc-575).

The thickness of the cortex and numbers of SATB2 $2^{+}, \mathrm{CTIP}^{+}, \mathrm{TBR} 1^{+}$, $\mathrm{p}-\mathrm{H} 3^{+}$, SOX2 $2^{+}$, and $\mathrm{TBR} 2^{+}$cells in $200-\mu \mathrm{m}$-wide columns, extending from the white matter to the pial surface of the somatosensory cortex, were manually determined using Imaris Software (Bitplane). For each animal, 3 sequential coronal sections, collected at $200-\mu \mathrm{m}$ intervals along the rostrocaudal axis, were quantified and averaged.

For histologic analyses, formalin-fixed brains were embedded in paraffin, and $4-\mu \mathrm{m}$ sagittal sections were cut, stained with Luxol fast blue, and counterstained with Nissl. Primary antibodies against NeuN (MilliporeSigma, MAB377B) and neurofilament (Covance, SMI-32R) were used for immunohistochemical analyses. Tissues were counterstained with hematoxylin. Cortical thickness and numbers of $\mathrm{NeuN}^{+}$

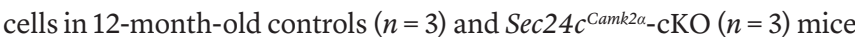
were determined using NIS-Elements (Nikon Instruments). For each animal, 3 sequential coronal sections at $50-\mu \mathrm{m}$ intervals were quantified and averaged.

TUNEL assay. TUNEL assays were performed with the ApopTag Kit (MilliporeSigma, S7110) according to the manufacturer's instructions. Low-magnification images were taken with a Marianas 2 microscope (Brandeis Life Sciences).

Real-time RT-PCR. Total RNA was extracted from mouse brains using the RNeasy Mini Kit (QIAGEN) according to the manufacturer's instructions. The reverse transcription reaction was performed using the SuperScript III First-Strand Synthesis Kit (Life Technologies, Thermo Fisher Scientific) according to the manufacturer's instructions. TaqMan gene expression assays consisting of FAMlabeled primer and probe sets specific for Sec24a (Mm00613960_ m1), Sec24b (Mm01313235_m1), Sec24c (Mm01269500_m1), Sec24d (Mm00660744_m1), and 18S were obtained from Applied Biosystems. Real-time RT-PCR reactions were performed in a total reaction volume of $25 \mu$ by using FastStart TaqMan Probe Master Reagent (Roche), and the results were analyzed using the ABI 7900 real-time PCR System (Applied Biosystems). Relative expression was normalized to $18 S$ RNA and calibrated to the respective controls.
To analyze ER stress, total RNA isolated from E13.5 cortices of control $(n=3)$ and $\operatorname{Sec} 24 c^{\text {Nes }}$-cKO $(n=3)$ animals was quantified by SYBR Green-based quantitative PCR, using the QuantStudio 3 Real-Time PCR System (Applied Biosystems). The following primers (from Thermo Fisher Scientific) were used $(53,54)$ : Atf4 (forward: 5'-ATGGCCGGCTATGGATGAT-3'; reverse: 5'-CGAAGTCAAACTCTTTCAGATCCATT-3'); Chop (forward: 5'-CTGCCTTTCACCTTGGAGAC-3'; reverse: 5'-CGTTTCCTGGGGATGAGATA-3'); Gadd34 (forward: 5'-GAGATTCCTCTAAAAGCTCGG-3'; reverse: 5 '-CAGGGACCTCGACGGCAGC-3'); Herpud1 (forward: 5'-GCAGTTGGAGTGTGAGTCG-3'; reverse: 5'-TCTGTGGATTCAGCACCCTTT-3'); BiP (forward: 5'-TGTGGTACCCACCAAGAAGTC-3'; reverse: 5'-TTCAGCTGTCACTCGGAGAAT-3'); sXbp1 (forward: 5'-GAGTCCGCAGCAGGTG-3'; reverse: 5'-GTGTCAGAGTCCATGGGA-3'); Edem1 (forward: 5'-GGGACCAAGAGGAAAAGTTTG-3'; reverse: 5'-GAGGTGAGCAGGTCAAATCAA-3'); ERdj4 (forward: 5'-CTTAGGTGTGCCAAAGTCTGC-3'; reverse: 5'-GGCATCCGAGAGTGTTTCATA-3'); and Gapdh (forward: 5'-AAGGCTGTGGGCAAAGTCATCC-3'; reverse: 5'-CTTCACCACCTTCTTGATGTCATC- $\left.3^{\prime}\right)$. Gapdh was used as an endogenous control for all reactions.

Immunoblot analyses. Tissues were lysed in Triton X-100based cell-lysis buffer. Proteins in cleared lysates were separated by electrophoresis on $4 \%$ to $12 \%$ Bis-Tris gels (Life Technologies, NP0335BOX) and transferred onto PVDF membranes. After incubation with a $5 \%$ skim milk block, blots were probed with antibodies against SEC24A (Proteintech, 15958-1-AP); SEC24B (Bethyl Laboratories, A304-876A-T); SEC24C (Abcam, ab122633 or Cell Signaling Technology, 8531); SEC24D (Cell Signaling Technology, 14687); actin (Santa Cruz Biotechnology, sc-1616-R); and GAPDH (MilliporeSigma, G9545). Membranes were then incubated with HRP-conjugated secondary antibodies and subjected to chemiluminescence detection (Amersham, RPN2232).

Electron microscopy. For transmission EM, E13.5 embryonic brains ( $n=1$ per genotype) were dissected in $0.05 \mathrm{M}$ phosphate buffer, followed by fixation in $2.5 \%$ glutaraldehyde and $2 \%$ PFA in $0.05 \mathrm{M}$ phosphate buffer. Coronal sections (100-mm) were cut with a vibratome and collected in cold PBS, rinsed in phosphate buffer, dehydrated through a series of graded ethanol-to-propylene oxide solutions, infiltrated and embedded in epoxy resin, and polymerized at $70^{\circ} \mathrm{C}$ overnight. Semi-thin $(0.5-\mu \mathrm{m})$ sections were stained with toluidine blue for light microscopic analysis. Ultra-thin (80$\mathrm{nm}$ ) sections were cut and imaged using an FEI Tecnai F 20 TEM FEG with an AT XR41 camera.

For scanning EM, tissues were fixed in $2.5 \%$ glutaraldehyde and $2 \%$ PFA in $0.05 \mathrm{M}$ phosphate buffer and rinsed in the same buffer. The tissue was stained using a modified heavy-metal-staining method (55) and processed through a graded series of alcohol and propylene oxide and then infiltrated in propylene oxide/epon gradients. The tissues were then infiltrated overnight in 100\% Epon 812 resin (Electron Microscopy Sciences, 14120) and polymerized for 48 hours in a $70^{\circ} \mathrm{C}$ oven. The samples were imaged with an FEI Helios G3 Scanning EM at $2 \mathrm{kV}, 0.4 \mathrm{nA}, 5 \times 5 \times 5 \mathrm{~nm}$ in column detector.

Behavioral tests. The elevated plus maze consists of 2 opposite open arms (11 in. $\times 2.5$ in.) intersected by 2 opposite "closed arms" (same as the open arms, but enclosed by 9 -inch walls) with a central open area ( $2.5 \mathrm{in} . \times 2.5 \mathrm{in}$.). The maze was elevated $22.5 \mathrm{in}$. above the ground. Each test began with the mouse being placed in the central 
area, facing an open arm. If the animal jumped off the maze, the trial was paused and the animal was placed back onto the center of the apparatus, facing an open arm. Each test lasted 15 minutes, and the following parameters were analyzed: time spent and distance traveled in the open arm, closed arm, and center.

The open field test arena (San Diego Instruments) consisted of four $16 \mathrm{in} . \times 16 \mathrm{in}$. clear plastic walls with a blue plastic floor, with white light conditions. Locomotor activity (total distance traveled and time spent in the center of the arena) was measured in 5-minute bins for 30 minutes. All trials were videotaped and analyzed using TopScan tracking software (Clever Sys Inc.).

Generation of human SEC24C ${ }^{-/}$iPSCs. BJFF.6 hiPSCs were reprogrammed from $\mathrm{BJ}$ foreskin fibroblasts using the Sendai virus as described previously (56). SEC24C $C^{-/-}$hiPSCs were generated using CRISPR-Cas9 technology. Briefly, BJFF.6 hiPSCs were transiently cotransfected with guide RNA (gRNA) expression plasmid (5'-GCTGGAGGTGCCCCCGAGGA-3'), Cas9 expression plasmid, and episomal GFP plasmid (Addgene, 27082) via nucleofection (Lonza, 4D-Nucleofector X-Unit) according to the manufacturer's protocol. Single cells were sorted by flow cytometry for transiently transfected cells $\left(\mathrm{GFP}^{+}\right)$into individual wells of 96-well plates coated with Vitronectin XF (STEMCELL Technologies) according to the manufacturer's recommended protocol. Sorted cells were plated into mTeSR media (STEMCELL Technologies) containing $1 \times$ CloneR supplement (STEMCELL Technologies). Cells were clonally expanded and verified for disruption of the endogenous locus via targeted deep sequencing to identify frameshift mutations. Two clones (1G6 and 2B9) were selected for further experiments.

Neuronal differentiation of hiPSCs. The hiPSCs were maintained with Essential 8 Flex Medium (Gibco, A2858501) in Matrigel-coated (Corning, 354277) culture vessels. Differentiation of hiPSCs into neurons was performed as described previously (46). Briefly, hiPSCs were seeded in Matrigel- and laminin- (Invitrogen, 23017-015) coated dishes and maintained in culture in chemically defined medium [DMEM/F12 (Gibco, 11330032): neurobasal (Gibco, 21103049)=1:1, $1 \times \mathrm{N} 2$ (Gibco, 17502048), 1×B27 (Gibco, 17504044), 1×nonessental amino acids (NEAA, Gibco, 11140050), 1\% GlutaMax (Gibco, 35050061), $2 \mu \mathrm{M}$ SB431542 (Stemgent, 04-0010-05), $2 \mu \mathrm{M}$ DMH1 (Tocris Bioscience, 4126), 1.4 $\mu \mathrm{M}$ CHIR99021 (Tocris Bioscience, 4423)] for 1 week. After 1 week of differentiation, the cells were mechanically dissociated (1:3 dilution) in the chemically defined medium containing $1 \mu \mathrm{g} / \mathrm{ml} \mathrm{SHH} \mathrm{C25II} \mathrm{(R \& D} \mathrm{Systems,} \mathrm{464-SH)}$ onto Matrigel- and laminin-coated plates. One week later, the cells were passed, as previously done in the chemically defined medium containing $1 \mu \mathrm{g} / \mathrm{ml} \mathrm{SHH} \mathrm{C25II} \mathrm{and} 10 \mathrm{ng} / \mathrm{ml} \mathrm{FGF} 4$ (R\&D Systems, 235-F4-025). After 1 week of culture in media containing SHH C25II and FGF4, the cells were seeded onto Matrigel- and laminin-coated dishes and maintained for 3 weeks in culture in a neuronal differentiation medium $(\mathrm{NDM})$ containing neurobasal medium, $1 \times \mathrm{N} 2,1 \times \mathrm{B} 27$, $1 \times$ NEAAs, $1 \%$ GlutaMax, $1 \mu \mathrm{g} / \mathrm{ml}$ laminin, $0.2 \mathrm{mM}$ vitamin C (Tocris Bioscience, 4055), $2.5 \mu \mathrm{M}$ DAPT (Tocris Bioscience, 2634), $10 \mathrm{ng} / \mathrm{ml}$ GDNF (PeproTech, 450-10 ), 10 ng/ml BDNF (PeproTech, 450-02), $10 \mathrm{ng} / \mathrm{ml}$ IGF-I (PeproTech, 100-11), and $1 \mathrm{ng} / \mathrm{ml}$ TGF- 33 (PeproTech, 100-36E). XTT cell viability assays were performed according to the manufacturer's protocol (Roche, 11465015001).

Immunocytochemistry and quantitative analysis. After 6 weeks of neuronal differentiation, cells growing on $35 \mu$-Dish (Ibidi,
81156) were fixed in $4 \%$ PFA, permeabilized with digitonin (100 $\mu \mathrm{g} / \mathrm{ml}$ in PBS), blocked in $1 \%$ BSA, and labeled with anti-TUJ1 (Covance, MMS-435P) and anti-CHOP antibodies. The cells were then incubated with Alexa Fluor-conjugated secondary antibodies (Invitrogen). The dishes were mounted in ProLong Gold Antifade Reagent with DAPI (Invitrogen). Images were acquired using a spinning-disc confocal Zeiss AxioObserver operated by a Marianas system (Intelligent Imaging Innovations) with a $\times 63$ oil objective. The TUJ1 $1^{+}$cell populations were counted among total cells labeled by DAPI. For each dish, at least 30 random fields were captured, and 3 dishes in each group were quantified manually. The intensity of nuclear CHOP from at least 30 images from each dish was analyzed using SlideBook 6 software (Intelligent Imaging Innovations). From the DAPI-stained images, the cells with bright, small, and dense DAPI ${ }^{+}$nuclei, as shown in Figure 7G, were categorized as condensed nuclei among the total DAPI-stained nuclei. At least 30 images and 3 dishes in each group were counted.

Statistics. All analyses were done blinded to the genotypes. All quantitative data are shown as the mean \pm SEM from 3 or more biological replicates. Statistical significance was determined by 2-way, repeated-measures ANOVA with Student-Neuman-Keuls post hoc analysis or a 2-tailed Student's $t$ test, as appropriate. A $P$ value of less than 0.05 was considered statistically significant.

Study approval. All animal experiments were reviewed and approved by the IACUC of St. Jude Children's Research Hospital.

\section{Author contributions}

BW and MK conceived and designed the experiments, analyzed the data, and wrote the manuscript. BW, JHJ, RM, BJWT, AK, ALW, and VPI performed the experiments. EJA, RK, and DG generated the $\operatorname{Sec} 24 c^{c-d}$ mice and edited the manuscript. STP and SMPM designed the strategy, generated the $S E C 24 C^{-/-}$hiPSC lines, and wrote the relevant section of the manuscript. SSZ and BJWT analyzed the behavioral data and wrote relevant sections of the manuscript. All authors reviewed the manuscript.

\section{Acknowledgments}

We thank Peter Vogel from the Veterinary Pathology Core (St. Jude Children's Research Hospital) and Victoria Frohlich, Sharon Frase, and Randall Wakefield from the Cell and Tissue Imaging Center (St. Jude Children's Research Hospital) for their technical assistance. We also thank Angela McArthur and Vani Shanker (St. Jude Children's Research Hospital) for editing the manuscript. This work was supported by grants from the National Heart, Lung, and Blood Institute (R01 HL114697, to MK) and the American Lebanese Syrian Associated Charities (ALSAC). RM was partially supported by a grant from the National Cancer Institute, NIH (R25CA23944).

Address correspondence to: Mondira Kundu, Department of Pathology, MS 343, St. Jude Children's Research Hospital, 262 Danny Thomas Place, Memphis, Tennessee 38105, USA. Phone: 901.595.6048; Email: Mondira.Kundu@stjude.org.

EJA's present address is: Human Oncology and Pathogenesis Program, Memorial Sloan Kettering Cancer Center, New York, New York, USA. 
1. Venditti R, Wilson C, De Matteis MA. Exiting the ER: what we know and what we don't. Trends Cell Biol. 2014;24(1):9-18.

2. Hunn BH, Cragg SJ, Bolam JP, Spillantini MG, Wade-Martins R. Impaired intracellular trafficking defines early Parkinson's disease. Trends Neurosci. 2015;38(3):178-188.

3. Zanetti G, Pahuja KB, Studer S, Shim S, Schekman R. COPII and the regulation of protein sorting in mammals. Nat Cell Biol. 2011;14(1):20-28.

4. Lee MC, Miller EA, Goldberg J, Orci L, Schekman R. Bi-directional protein transport between the ER and Golgi. Annu Rev Cell Dev Biol. 2004;20:87-123.

5. Jensen D, Schekman R. COPII-mediated vesicle formation at a glance. JCell Sci. 2011;124(Pt 1):1-4

6. Roberg KJ, Crotwell M, Espenshade P, Gimeno $\mathrm{R}$, Kaiser CA. LST1 is a SEC24 homologue used for selective export of the plasma membrane ATPase from the endoplasmic reticulum. JCell Biol. 1999;145(4):659-672.

7. Higashio H, Kimata Y, Kiriyama T, Hirata A, Kohno K. Sfb2p, a yeast protein related to Sec24p, can function as a constituent of COPII coats required for vesicle budding from the endoplasmic reticulum. J Biol Chem. 2000;275(23):17900-17908.

8. Tang BL, Kausalya J, Low DY, Lock ML, Hong W. A family of mammalian proteins homologous to yeast Sec24p. Biochem Biophys Res Commun. 1999;258(3):679-684.

9. Boyadjiev SA, et al. Cranio-lenticulo-sutural dysplasia is caused by a SEC23A mutation leading to abnormal endoplasmic-reticulum-to-Golgi trafficking. Nat Genet. 2006;38(10):1192-1197.

10. Boyadjiev SA, et al. Cranio-lenticulo-sutural dysplasia associated with defects in collagen secretion. Clin Genet. 2011;80(2):169-176.

11. Schwarz K, et al. Mutations affecting the secretory COPII coat component SEC23B cause congenital dyserythropoietic anemia type II. Nat Genet. 2009;41(8):936-940.

12. Lang MR, Lapierre LA, Frotscher M, Goldenring JR, Knapik EW. Secretory COPII coat component Sec23a is essential for craniofacial chondrocyte maturation. Nat Genet. 2006;38(10):1198-1203.

13. Zhu M, et al. Neural tube opening and abnormal extraembryonic membrane development in SEC23A deficient mice. Sci Rep. 2015;5:15471.

14. Tao J, et al. SEC23B is required for the maintenance of murine professional secretory tissues. Proc Natl Acad Sci U S A. 2012;109(29):E2001-E2009.

15. Peng R, De Antoni A, Gallwitz D. Evidence for overlapping and distinct functions in protein transport of coat protein Sec $24 \mathrm{p}$ family members. J Biol Chem. 2000;275(15):11521-11528.

16. Miller E, Antonny B, Hamamoto S, Schekman R. Cargo selection into COPII vesicles is driven by the Sec24p subunit. EMBOJ. 2002;21(22):6105-6113.

17. Miller EA, et al. Multiple cargo binding sites on the COPII subunit Sec24p ensure capture of diverse membrane proteins into transport vesicles. Cell. 2003;114(4):497-509.

18. Mossessova E, Bickford LC, Goldberg J. SNARE selectivity of the COPII coat. Cell. 2003;114(4):483-495.

19. Matsui K, Furukawa S, Shibasaki H, Kikuchi T. Reduction of nerve growth factor level in the brain of genetically ataxic mice (weaver, reeler). FEBS Lett. 1990;276(1-2):78-80.
20. Russell C, Stagg SM. New insights into the structural mechanisms of the COPII coat. Traffic. 2010;11(3):303-310.

21. Wendeler MW, Paccaud JP, Hauri HP. Role of Sec24 isoforms in selective export of membrane proteins from the endoplasmic reticulum. $E M B O$ Rep. 2007;8(3):258-264.

22. Mancias JD, Goldberg J. Structural basis of cargo membrane protein discrimination by the human COPII coat machinery. EMBO J. 2008;27(21):2918-2928.

23. Farhan H, Reiterer V, Korkhov VM, Schmid JA, Freissmuth M, Sitte HH. Concentrative export from the endoplasmic reticulum of the gammaaminobutyric acid transporter 1 requires binding to SEC24D. J Biol Chem. 2007;282(10):7679-7689.

24. Sucic $S$, et al. The serotonin transporter is an exclusive client of the coat protein complex II (COPII) component SEC24C. JBiol Chem . 2011;286(18):16482-16490.

25. Chen XW, et al. SEC24A deficiency lowers plasma cholesterol through reduced PCSK9 secretion. Elife. 2013;2:e00444.

26. Yang XY, et al. Mutations in the COPII vesicle component gene SEC24B are associated with human neural tube defects. Hum Mutat. 2013;34(8):1094-1101.

27. Merte J, et al. Sec 24 b selectively sorts Vangl2 to regulate planar cell polarity during neural tube closure. Nat Cell Biol. 2010;12(1):41-46; sup pp 1.

28. Garbes L, et al. Mutations in SEC24D, encoding a component of the COPII machinery, cause a syndromic form of osteogenesis imperfecta. Am J Hum Genet. 2015;96(3):432-439.

29. Moosa S, et al. Mutations in SEC24D cause autosomal recessive osteogenesis imperfecta. Clin Genet. 2016;89(4):517-519.

30. Zhang $\mathrm{H}$, et al. Novel mutations in the SEC24D gene in Chinese families with autosomal recessive osteogenesis imperfecta. Osteoporos Int. 2017;28(4):1473-1480.

31. Sarmah S, Barrallo-Gimeno A, Melville DB, Topczewski J, Solnica-Krezel L, Knapik EW. Sec24D-dependent transport of extracellular matrix proteins is required for zebrafish skeletal morphogenesis. PLoS One. 2010;5(4):e10367.

32. Adams EJ, Chen XW, O'Shea KS, Ginsburg D. Mammalian COPII coat component SEC24C is required for embryonic development in mice. J Biol Chem . 2014;289(30):20858-20870.

33. Baines AC, Adams EJ, Zhang B, Ginsburg D. Disruption of the Sec24d gene results in early embryonic lethality in the mouse. PLoS One. 2013;8(4):e61114.

34. Adams EJJ, et al. Murine SEC24D Can Substitute Functionally for SEC24C in vivo. bioRxiv. https://www.biorxiv.org/content/ early/2018/03/22/284398. Accessed May 17, 2018.

35. Tronche F, et al. Disruption of the glucocorticoid receptor gene in the nervous system results in reduced anxiety. Nat Genet. 1999;23(1):99-103.

36. Molyneaux BJ, Arlotta P, Menezes JR, Macklis JD. Neuronal subtype specification in the cerebral cortex. Nat Rev Neurosci. 2007;8(6):427-437.

37. Tsien JZ, et al. Subregion- and cell type-restricted gene knockout in mouse brain. Cell. 1996;87(7):1317-1326.

38. Walf AA, Frye CA. The use of the elevated plus maze as an assay of anxiety-related behavior in rodents. Nat Protoc. 2007;2(2):322-328.

39. Lein ES, et al. Genome-wide atlas of gene expression in the adult mouse brain. Nature. 2007;445(7124):168-176

40. Tabas I, Ron D. Integrating the mechanisms of apoptosis induced by endoplasmic reticulum stress. Nat Cell Biol. 2011;13(3):184-190.

41. Walter P, Ron D. The unfolded protein response: from stress pathway to homeostatic regulation. Science. 2011;334(6059):1081-1086.

42. Yoshida H, Matsui T, Yamamoto A, Okada T, Mori K. XBP1 mRNA is induced by ATF6 and spliced by IRE1 in response to ER stress to produce a highly active transcription factor. Cell.2001;107(7):881-891.

43. Yoshida H, Matsui T, Hosokawa N, Kaufman RJ, Nagata K, Mori K. A time-dependent phase shift in the mammalian unfolded protein response. Dev Cell. 2003;4(2):265-271.

44. Lee AH, Iwakoshi NN, Glimcher LH. XBP-1 regulates a subset of endoplasmic reticulum resident chaperone genes in the unfolded protein response. Mol Cell Biol. 2003;23(21):7448-7459.

45. Silva RM, et al. CHOP/GADD153 is a mediator of apoptotic death in substantia nigra dopamine neurons in an in vivo neurotoxin model of parkinsonism. J Neurochem. 2005;95(4):974-986.

46. Lu J, et al. Generation of serotonin neurons from human pluripotent stem cells. Nat Biotechnol. 2016;34(1):89-94.

47. Nakagawa T, et al. Caspase-12 mediates endoplasmic-reticulum-specific apoptosis and cytotoxicity by amyloid-beta. Nature. 2000;403(6765):98-103.

48. Visel A, Thaller C, Eichele G. GenePaint.org: an atlas of gene expression patterns in the mouse embryo. Nucleic Acids Res. 2004;32(Database issue):D552-D556.

49. Molyneaux BJ, et al. DeCoN: genome-wide analysis of in vivo transcriptional dynamics during pyramidal neuron fate selection in neocortex. Neuron. 2015;85(2):275-288.

50. Holmes A, Yang RJ, Lesch KP, Crawley JN, Murphy DL. Mice lacking the serotonin transporter exhibit 5-HT(1A) receptor-mediated abnormalities in tests for anxiety-like behavior. Neuropsychopharmacology. 2003;28(12):2077-2088.

51. Erbay E, et al. Reducing endoplasmic reticulum stress through a macrophage lipid chaperone alleviates atherosclerosis. Nat Med. 2009;15(12):1383-1391.

52. Ozcan L, et al. Endoplasmic reticulum stress plays a central role in development of leptin resistance. Cell Metab. 2009;9(1):35-51.

53. Rutkowski DT, et al. Adaptation to ER stress is mediated by differential stabilities of pro-survival and pro-apoptotic mRNAs and proteins. PLoS Biol. 2006;4(11):e374.

54. Sun S, et al. IRE1 $\alpha$ is an endogenous substrate of endoplasmic-reticulum-associated degradation. Nat Cell Biol. 2015;17(12):1546-1555.

55. Deerinck T, Bushong E, Lev-Ram V, Shu X, Tsien R, Ellisman M. Enhancing serial block-face scanning electron microscopy to enable high resolution 3-D nanohistology of cells and tissues. Microsc Microanal. 2010;16(S2):1138-1139.

56. Fusaki N, Ban H, Nishiyama A, Saeki K, Hasegawa M. Efficient induction of transgene-free human pluripotent stem cells using a vector based on Sendai virus, an RNA virus that does not integrate into the host genome. Proc Jpn Acad, Ser B, Phys Biol Sci. 2009;85(8):348-362. 